\title{
EBDOMADARI VERSUS CANONICI: GLI ISTITUTI CLERICALI, IL POTERE ECCLESIALE E LA TOPOGRAFIA MEDIEVALE DEL COMPLESSO EPISCOPALE DI NAPOLI
}

\author{
Università degli Studi di Napoli Federico II
}

Riassunto: In questo articolo si propone una nuova interpretazione di una serie di documenti medievali (X-XIV secolo) menzionanti le congregazioni clericali della Chiesa cattedrale napoletana, la congregatio ecclesiae Sanctae Restitutae e la congregatio Salvatoris. Le conclusioni a cui si giunge è che non si tratta di due Capitoli di Canonici facenti capo a due distinti edifici cattedrali, Santa Restituta e il Salvatore, ma di due congregazioni gerarchicamente differenziate all'interno della medesima Cattedrale, i membri di una delle quali, ufficialmente chiamati dal 1337 Ebdomadari, inventarono nel Settecento, per ragioni di denaro e di potere, la tradizione di un seconda antica Cattedrale.

Parole chiave: Cattedrale di Napoli;Topografia medievale; Congregazioni; Canonici del Capitolo; Ebdomadari.

\begin{abstract}
This article suggests a new interpretation of a series of medieval records (X-XIV centuries), in which the congregatio ecclesiae Sanctae Restitutae and the congregatio Salvatoris, are mentioned, both congregations of priests attached to Naples Cathedral. The authoress reaches the conclusion that these were not two chapters of canons corresponding to two different cathedral buildings: Santa Restituta and the Salvatore, but rather two hierarchically different congregations within the same Cathedral. The members on one of these congregations -from 1337 officially called Ebdomadari-, in the 18th century, invented the tradition of a second old Cathedral, for economic reasons and to increase their power.
\end{abstract}

Keywords: Naples'Cathedral; Medieval Topography; Congregations; Chapter Canons; Ebdomadari.

\section{SOMMARIO}

1. Un inedito antefatto. 2. Una questione di lunga durata. 3. I documenti. 4. Falsificazioni e fraintendimenti. 5. Nuove proposte.

\section{UN INEDITO ANTEFATTO}

Nel 1772, si dava alle stampe a Napoli un poderoso volume dal titolo Memorie in difesa dell'insigne collegio dei sacri ministri della Cattedrale 
napoletana, chiamati Eddomadari ${ }^{1}$, all'interno del quale l'anonimo autore accludeva una carta di tavola ripiegata in quattro parti (fig. 1) ${ }^{2}$.

Una lunga didascalia correda l'immagine raffigurata nella tavola: "Processione generale del ricevimento del cardinal Barberino legato a latere della Santità di Clemente XI alla Maestà di Filippo V, fatta a 29 marzo 1702, nella quale non intervenne il Capitolo del Duomo il quale, dopo aver ricevuto ed ossequiato il cardinal legato a Santa Maria a Cappella, si spogliò e se n'andò privatamente al Duomo per ivi ricevere esso legato, e si osservano i soli Eddomadari precedere la Collegiata di San Giovanni Maggiore".

La rappresentazione che si squaderna davanti al lettore è piuttosto singolare: su cavalli maestosi incedono il re di Spagna, Filippo V, e il legato pontificio, Carlo Barberini, protetti da aerei baldacchini, scortati da paggi e palafrenieri, e accompagnati da illustri membri dei loro seguiti, cardinali, vicerè, baroni, pur essi a cavallo. Davanti a loro, a piedi, avanzano composti gli Ebdomadari della Cattedrale, i preti cosiddetti "Quaranta" e gli allievi del Seminario, tutti preceduti dalla "croce degli Eddomadari"; più avanti ancora ci sono i membri della Collegiata di San Giovanni Maggiore, i parroci, il clero secolare, e i rappresentanti delle quattro maggiori parrocchie cittadine, preceduti dalle rispettive croci. I personaggi della processione, la cui corretta identificazione è consentita da lettere maiuscole poste loro accanto, camminano su una zolla di terra appena accennata, priva di ulteriori connotazioni distintive, e si stagliano con contorni netti sul fondale monocromo della pagina, senza alcuna scenografia di cornice.

L'immagine si propone come una trascrizione asciutta, sintetica e abbreviata, ma non per questo meno suggestiva e incuriosente, di un episodio avvenuto settanta anni prima, durante il soggiorno a Napoli di Filippo V, durato dal 17 aprile al 2 giugno del $1702^{3}$, un periodo di ininterrotti festeggiamenti che ebbe il suo acme il 20 maggio, in una cavalcata di cui furono protagoniste al fianco del re migliaia di persone, tra cui novemila soldati, centosessanta titolati e cavalieri, tre cardinali, qualche decina di vescovi e arcivescovi. L'anziano cardinale Barberini ${ }^{4}$, inviato dal papa Clemente XI come legato a latere, approdato sulle coste campane il 22 maggio, incontrò solennemente il re il 29 maggio (la didascalia dell'incisione dice erroneamente

${ }^{1}$ Il volume non reca sul frontespizio né l'indicazione dell'autore né quella dell'editore. Da un riferimento interno si deduce che esso fu redatto da don Francesco Peccheneda, prolifico e abile Presidente della Regia Camera di Napoli, sul quale si veda Camillo MINIERI RICCIO, Memorie storiche degli scrittori nati nel Regno di Napoti, Tipografia dell'Aquila, Napoli, 1844, p. 262 e p. 409.

${ }^{2}$ La Biblioteca della Società Napoletana di Storia Patria possiede diversi esemplari di questo volume (ne ho potuti consultare tre), tutti corredati della carta di tavola. Ringrazio la direzione della Biblioteca per avermi consentito di riprodurre l'incisione.

${ }^{3}$ Giuseppe GALASSO, Napoli nel Viceregno spagnolo 1696-1707, in Storia di Napoli. VII. Dal Viceregno alla Repubblica del '99, Società Editrice Storia dì Napoli, Napoli, 1972, in part. pp. 239-264; IDEM, Napoli spagnola dopo Masaniello: politica cultura e società, 2 voll., Sansoni, Firenze, 1982.

${ }^{4}$ Alberto Merola, ad vocem Barberini, Carlo, in Dizionario Biografico degli Italiani, VI, Istituto dell'Enciclopedia Italiana, Roma, 1964, pp. 171-172. 
"marzo"), in una cerimonia molto affollata e sfarzosa che si concluse con una seconda cavalcata, non meno spettacolare della prima.

Se la visita del re di Spagna fu uno degli eventi più memorabili dell'intero Settecento napoletano, perché i Napoletani non vedevano la faccia di un loro sovrano da centossessantasei anni, quando era giunto Carlo $\mathrm{V}^{5}$, l'arrivo di Barberini con il suo seguito di più di trecento uomini, tra alti prelati, nobili romani, servi e assistenti, fu un evento nell'evento, per le molte implicazioni politiche che portava con sé. Di esso resta vivida memoria, tra l'altro, in una lettera di Antonio Bulifon ${ }^{6}$, e in una descrizione di Francesco Bianchini, riedita da Pietro Ercole Visconti nel $1858^{7}$.

Ma che senso e che scopo poteva avere, a quasi un secolo di distanza, rievocare visivamente quei fatti e quei personaggi? E perché nel 1772 si inseriva la raffigurazione di un episodio accaduto nel 1702 in un testo che con i fasti della monarchia spagnola non aveva alcun legame?

Le immagini parlano, quel che comunicano non può essere ignorato e, a guardar bene, c'è nella scena qualcosa che non funziona: mancano infatti i Canonici del Capitolo cattedrale, che secondo la didascalia se ne erano andati in anticipo, e i primi ad avanzare, proprio davanti ai vicerè e ai baroni del Regno, sono invece gli Ebdomadari, il corpo di mansionari inferiori sottoposti al Capitolo e tenuti alla celebrazione quotidiana del servizio liturgico. E se si osserva con attenzione, di tutto il clero effigiato gli Ebdomadari sono gli unici ad avere dimensioni fisiche pari solo a quelle dei membri dell'aristocrazia cardinalizia e regnicola, mentre gli altri ecclesiastici, persino i potenti membri della Collegiata di San Giovanni Maggiore, che godevano di grande autorevolezza, sono di dimensioni nettamente inferiori.

Chi ha fatto incidere questa solenne processione di nobili e di chierici, nella quale gli Ebdomadari della Cattedrale occupano il primo posto, ha scelto di mettere in scena soltanto un frammento della cavalcata solenne del cardinale Barberini, isolandolo e ritagliandolo da rappresentazioni contempo-

\footnotetext{
${ }^{5}$ G. GALASSO, Napoli nel Viceregno cit., p. 247.

${ }^{6}$ Antonio BULIFON, Altra lettera scritta da Antonio Bulifon a un suo amico: nella quale gli dà ragguaglio della seconda cavalcata fatta in Napoli per la solenne entrata dell'eminentissimo signor Cardinale Carlo Barberini, mandato da Sua Santità in qualità di suo legato a latere di Filippo V monarca delle Spagne, nella stamperia di Felice Mosca, in Napoli, 1702. Sul medesimo argomento si vedano anche: Distinto racconto della real cavalcata fatta da'titolati, cavalieri e baroni di questo regno, tra' quali tre cardinali, molti prencipi e signori romani per l'ingresso in questa fedelissima citta di Napoli del nostro gloriosissimo monarca Filippo Quinto, per Giovanni Rosselli stampatore, in Napoli, 1702; [D. Franciscus Muscettola, Dux Spezzani] Molis et inscriptionum Sedilis Montaneae, die vicesima maii anni 1792, qua pubblice Neapolim ingressus est Philippus $V$, Rex noster optimus maximus narratiuncula, Typis Antonii Solofrano, Neapoli, MDCCII.

${ }^{7}$ Francesco BIANCHINI, Lettera ad un amico in ragguaglio della legazione dell'eminentissimo e reverendissimo signor Cardinale Carlo Barberini alla Maestà cattolica del Re Filippo V in nome del Sommo Pontefice Clemente XI, l'anno 1702 , per Pietro Olivieri, in Roma, 1702 ;
Descrizione della solenne legazione del Cardinale Carlo Barberini e Filippo V, nuovamente Descrizione della solenne legazione del Cardinale Carlo Barberini e Filippo V, nuovamente
posta in luce nella faustissima esaltazione della sacra porpora dell'eminentissimo signor
Cardinale Giuseppe Milesi Pironi Ferretti per cura del commendatore P. E. Visconti commissario delle antichità, Tipografia delle Belle Arti, Roma, 1858.
} 
ranee che quell'evento illustravano compiutamente, quale ad esempio la carta di tavola a corredo della Distinta e sincera relazione della regal cavalcata fatta per il pubblico ingresso in questa città di Napoli del gloriosissimo nostro monarca Filippo Quinto (fig. 2) ${ }^{8}$. Ma se si pongono a confronto le due scene e soprattutto se si leggono le relazioni e i resoconti del 1702, ci si accorge subito che l'incisione del 1772 non corrisponde al vero.

Chi ha operato questa selezione sui memorabili accadimenti dell'inizio del secolo ha voluto mettere in risalto la dignità e il primato di un collegio clericale che non era in alcun modo il primo collegio cittadino, essendo la diocesi rappresentata al più alto grado esclusivamente dall'arcivescovo e dai Canonici del Capitolo cattedrale. Chi ha eseguito il disegno, scontornando e ingrandendo il piccolo particolare degli Ebdomadari che in quella miriade di corpi non erano che un puntino sullo sfondo, deve esser stato incaricato di cercare un evento il più rappresentativo possibile per dichiarare visivamente un concetto falso: che i primi chierici della Capitale del Viceregno, gli unici degni di precedere immediatamente i cavalli del re e del legato del papa, erano gli Ebdomadari della Cattedrale di Napoli.

\section{UNA QUESTIONE DI LUNGA DURATA}

La Cattedrale di Napoli (fig. 3), a tutt'oggi sede dell'arcivescovo, è ancora fruibile, malgrado le trasformazioni di età moderna ${ }^{9}$, nelle forme monumentali che le furono attribuite tra la fine del Duecento e i primi decenni del Trecento ${ }^{10}$. Il più antico documento riguardante la sua costruzione risale al 16 giugno del 1294: in esso, Carlo II d'Angiò, "rex Siciliae", in risposta ad una supplica rivoltagli dall'arcivescovo Filippo Minutolo, "dilectus familiaris et fidelis", dava ordine al Capitano della città di stimare il valore

\footnotetext{
${ }^{8}$ Distinta e sincera relazione della regal cavalcata fatta per il pubblico ingresso in questa fedelissima città di Napoli dal nostro monarca Filippo Quinto da' titolati cavalieri e baroni di questo Regno, tra' quali tre cardinali, molti prencipi e signori romani, con tutte le sollennità che in funzioni cosi grandi accostumansi, seguita il di 20 maggio 1702 , per Domenico Antonio
Parrino e Cavallo Michele Luigi Mutio, in Napoli, 1702 . L'incisione qui riprodotta è la seconda delle due incisioni che accompagnavano il breve testo: la prima rappresenta appunto la cavalcata del 20 maggio.

${ }^{9}$ Per le modifiche subite dall'edificio nel corso dei secoli: Roberto DI STEFANO Franco STRAZZULLO, Restauri e scoperte nella Cattedrale di Napoli " "Napoli Nobilissima", İV serie, $\mathrm{X}(1971), \mathrm{pp}$. 3-59; R. DI STEFANO, La Cattedrale di Napoli. Storia restauro,' scoperte, ritrovamenti con documenti per la storia dei restauri a cura di F Strazzullo, Editoriale Fondazione P. Corsicato, Napoli, 1991.

${ }^{10}$ Per un'approfondita disamina dei caratteri architettonici dell'edificio: Caroline BRUZELIUS, Ipotesi e proposte sul Duomo di. Napoli, in Il Duomo di Napoli dal paleocristiano all'eta angioina. Atti della I Giornata di Studi su Napoli (Losanna, 23 novembre 2000), a cura di Serena ROMANO e Nicolas BOCK, Electa Napoli, Napoli, 2002, pp. 119-131; C. BRUZELIUS, Le pietre di Napoli. L'architettura religiosa nell'Italia angioina, 1266-1343, Viella, Roma, 2005 , in part. pp. 96 ss.
} 
di un terreno necessario all'edificazione della fabbrica ${ }^{11}$. Le carte d'archivio non consentono di risalire più indietro di questa data per stabilire la cronologia dell'edificio ${ }^{12}$.

Se all'esterno, sullo scenario cittadino, la fondazione della nuova cattedrale si configurò come uno dei fulcri della politica architettonica e funeraria della dinastia reale angioina ${ }^{13}$, all'interno, nell'ambito chiuso e protetto del complesso episcopale, innescò un processo di codificazione delle norme liturgiche e di ridefinizione degli istituti clericali destinato ad avere, sulla lunga durata, un peso considerevole nelle vicende dell'episcopato napoletano. Promotori di questo processo furono due potenti prelati, Humbert d'Ormont (1308-1320) e Giovanni Orsini (1328-1358) ${ }^{14}$, ma protagoniste furono le due maggiori congregazioni della Cattedrale: i Canonici del Capitolo e il corpo di mansionari chiamati Ebdomadari: i primi attestati come tali dalla metà del XII secolo, mentre i secondi solo dal 1337, come avremo modo di vedere più avanti.

$\grave{E}$ opinione diffusa e indiscussa che i Canonici e gli Ebdomadari siano gli eredi di due precedenti congregazioni, il cui nome ricorre nei documenti a partire dal X secolo, la congregazione di Santa Restituta e la congregazione del Salvatore o della Stefania, da tutti ritenute essere due distinti capitoli di due diverse antiche cattedrali: l'una, da riconoscersi nella basilica di Santa Restituta, tuttora praticabile come cappella laterale dell'edificio gotico; l'altra, intitolata al Salvatore e denominata Stefania, eretta su un asse parallelo a quello di Santa Restituta e coincidente con il transetto dell'edificio gotico, ritenuta distrutta proprio nel corso dei lavori di costruzione della nuova cattedrale ${ }^{15}$

L'identificazione dei due collegi e l'interpretazione dei documenti che li riguardano sono però estremamente più complesse di quanto appaiano ad un primo sguardo. Per sciogliere questo nodo, che negli ultimi tre secoli si è via

${ }^{11}$ Il documento si trova trascritto in Biagio CANTERA, L'edificazione del Duomo di Napoli al tempo degli Angioini, Società Tipografica Editrice Bartolo Longo, Valle di Pompei, 1890, p. 7 e nota 3.

${ }^{12} \mathrm{Su}$ tale questione rinvio al mio articolo La Cappella di San Ludovico nella Cattedrale di Napoli, le sepolture dei sovrani angioini, le due statue dei re e gli errori della tradizione storiografica moderna, in corso di stampa sulla "Zeitschrift für Kunstgeschichte", 2007, 70.

${ }^{13}$ Sull'immagine di Napoli ad inizio Trecento: Rosalba DI MEGLIO, Napoli 1308: una città cantiere, "Archivio Storico per le Province Napoletane", CXXIII (2005), pp. 93-113.

${ }^{14}$ Per le vicende riguardanti i due arcivescovi: Bartolomeo CHIOCCARELlo, Antistitum praeclarissimae Neapolitanae Ecclesiae catalogus ab apostolorum temporibus ad hanc nostram aetatem et ad annum 1643, typis Francisci Savil, expensis Petri Agnelli Porrini, Neapoli, 1643 , $\mathrm{pp}$. $197 \mathrm{ss}$. e pp. $220 \mathrm{ss}$. Sul ruolo di Humbert d'Ormont come committente di opere d'arte: Tanja MICHALSKY (Hg.), Medien der Macht. Kunst zur Zeit der Anjous in Italien, Reimer, Berlin, 2001, pp. 191-224; S. ROMANO, La cattedrale di Napoli, i vescovi e l'immagine. Una storia di lunga durata, in Il Duomo di Napoli dal paleocristiano all' età angioina cit., pp. 7-20.

${ }^{15}$ Tra i numerosi interventi novecenteschi nei quali quest'assunto si dà per certo, esemplari sono le pagine di F. STRAZZULLO, Le due cattedrali di Napoli e la "Dissertatio historica" del Mazzocchl, "Archivio storico di Terra di Lavoro", IV (1965-1975), pp. 321-327, in part. p. 323: "Ma come si può sostenere che la Stefania era la stessa basilica di Santa Restituta, quando i documenti attestano l'esistenza di una doppia congregatio: una congregatio sacerdotum et clericorum ecclesiae S. Restitutae, distinta da una congregatio Stephanie? Quindi due chiese!". 
via più intricato, si rende pertanto necessario attraversare fatti ed episodi che si dispiegano su circa dieci secoli, in una sorta di corsa ad ostacoli tra ciò che è materialmente documentato e ciò che, sebbene falso e inventato, è diventato convinzione comune. Molto lavoro c'è ancora da fare al riguardo e le ipotesi che qui si propongono costituiscono solo uno stadio di un'indagine ad ampio raggio, che di continuo obbliga a superare i confini esistenti tra le discipline. La storia dell'architettura napoletana del Medioevo, infatti, non è solo una storia di pietre, ma è anche una storia di parole, di idee, di immagini, e soprattutto di tradizioni.

\section{I DOCUMENTI}

Tra le carte d'archivio pubblicate nel corso dell'età moderna da quella schiera di storici e eruditi locali ai quali si deve la conservazione della memoria del Medioevo meridionale, ve ne sono alcune che registrano, in connessione con la Sede episcopale, una congregatio ecclesiae Stephaniae, una congregatio Sanctae Restitutae e una congregatio Salvatoris, altre invece che nominano espressamente il Capitolo dei Canonici della Chiesa cattedrale ${ }^{16}$.

I più antichi di questi documenti sono l'uno dell'11 novembre $932 \mathrm{e}$ l'altro del 2 febbraio 977. Il primo dei due è un testamento: un certo Sergio, figlio di Costantino, dando disposizioni sulla divisione dei suoi beni, nel considerare il caso che i suoi eredi restino in futuro senza eredi, destina una porzione del suo patrimonio alla "congregatio chartulae ecclesiae Stephaniae" 17 . Il secondo è uno strumento di vendita: un certo Pietro, figlio

\footnotetext{
${ }^{16}$ La documentazione riguardante le congregazioni, emerșa nel corso dei secoli dagli archivi napoletani e più volte pubblicata in ordine sparso dagli eruditi locali (a cominciare da Chioccarello), fu raccolta e edita in ordinata sequenza cronologica dal canonico napoletano Pasquale SANTAMARIA, nella sua Historia Collegii Patrum Canonicorum Metropolitanae Ecclesiae Neapolitanae, ex Typis Francisci Jannini et filiorum, Neapoli 1900. La maggior parte dei documenti oggetto della mia indagine, riportati infra alle note 17-34, sono stati qui parte dei documenti oggetto della mia indagine, riportati infra alle note 17-34, sono stati qui trascritti sulla base di Santamaria senza apportare variazioni redazionali, fatti salvi lo scioglimento delle abbreviazioni, l'inserimento delle maiusçole e qualche modificazione della punteggiatura, laddove necessari ad una migliore comprensione. Si è scelto di trascriverli per intero, così come pubblicati dall'erudito canọnico all'inizio del Novecento, sia perché il volume è raramente rintracciabile fưori dạ Napoli, sia per consentirne immediątamente una più efficace lettura e interpretazione. Si è poi scelto di nọ segnalare con il "sic" la presenza di evident errori, in quanto l'edizione di Santamaria ne è cosi ricca (vuoi per responsabilità del curatore vuoi per lo stato di conservazione dei documenti) che gli interventi del trascrittore sarebbero stati ẹccessivi. I più significativi riferimenti alle congregazioni e alla Chiesa di Napoli presenti nei singoli documenti saranno evidenziati in corsivo. Nelle citazioni nel testo dell articolo saranno sempre ripristinati il dittongo "ae" e la terminazione in "is" del genitivo, anche quando assenti nell'edizione di Santamaria.

17 “Die 11 mensis novembris, indictione VI, imperante domino noștro Constantino magno imperatore annis 25, sed et Romano et Christophoro eius filio magnis imperatoris annis 12. Dispositum factum a Sergio filio quondam Constantini et quondam domine, iugalium, cum voluntate praesentis Blactu, honestae feminae coniugis suae, ut qualiter inferius iudicaberit de omni sua substantia firmum et stabile maneat in perpetuum Et primum omnium disponit, od suum ovitum Iohannes filius quondam Gregoriipentum. Et primum omnium isponit, ut ad suum ovitum lohannes filius quondam Gregorii magnifici et Aligernus filius item domin Aligerni et Petrus filius quondam Leonis vendant integram portionem suam, quam ei tetigit de terras positas foris gripta et caballum suum, et de pretio, quod exinde tulerint, dent solidos 4 ad Aligernum et Annam germanos bernaculos suos; reliquos autem distribuant pro anima sua per sacerdotes et pauperes fratres Christi, uti ipsi praeviderint. Item legat eisdem bernaculis integram petiam de terra, quae vocatur rotundula, sita ad Baccilianum, cum omnibus sibi
} 
di Giuliano, vende a Stefano, figlio di Leone, un fondo in località San Pietro a Patierno: nel redigere l'atto di vendita si stabilisce che, se richiesto dal compratore, il venditore debba mostrare sia la carta di acquisto del terreno che suo padre aveva ricevuto da Marino Aurifice, sia un'altra "chartula securitatis" che lo stesso Giuliano aveva avuto da Cesareo, presbitero e cimiliarca della Chiesa napoletana, e da tutta la "congregatio chartulae ecclesiae Stephaniae" 18 .

pertinentibus, ut dividant illam inter se equaliter in eo tamen tenore ut, si quis eorum infra etatem aut sine herede obierit, alteri, qui ex eis supervixerit, eiusque heredibus detur portio eius, si infra aetatem, gratis: si vero legitimus, ad medietatem pretii, quo appretiata fuerit a christianissimis viris, per manu cui disposuerit et, si non disposuerit, per manu cimiliarcha, qui tunc tempore fuerit in Sancta Neapolitana Ecclesia. Si vero ambo infra etatem aut sine herede obierint, succedat eisdem Ursus filius suus eiusque herede eadem lege, ut supra. Omnem vero hereditatem suam seu substantiam, de intus et foris, domos et casales, terras sationales per hortua vel montibus, res mobiles vel immobiles seseque moventes, omnesque sibi pertinentes relinquit Urso filio suo, ita ut, si infra etatem vel sine herede obierit, omnes hospites sui et fundora vivorum et mortuorum, seu commenditi cum cespitibus et consuetudinibus cum uno modio de terra, ubi ipse sibi eligere voluerit, sint de quodam Urso germano suo eiusque modio de terra, ubi ipse sibi eligere voluerit, sint de quodam Urso germano suo eiusque fuerint vel illorum heredes, et dividant sibi illud inter se equaliter in eo tenore, ut unus ex eis alteri, qui infra etatem aut sine herede obierit, succedat: et, si ad ovitum ipsius Ursi, praedict bernaculi non fuerint, aut ipsi infra etatem aut sine heredibus obierint, legat modium unum de terra ad Paternum ecclesiae Sancti Petri ad Paternum, et reliqua congregationi chartulae ecclesiae Stephaniae. Item disponit ut, qui de memoratis bernaculis aut eorum heredibus aliquod de portione sua dare habuerit, nulli alie persone dare presumat, nisi inter se eorumque heredibus, et in pretio, sicuti appretiatum fuerit et si inter se tollere noluerint, vendant ipsi fillio suo et, si iste aut heredes eius emere noluerint, cui dare voluerint, in eorum sit potestate. Insuper disponit quod Blactu honesta femina, coniux sua, sit domna et domina in omni sua hereditate regendi, gubernandi et fruendi eam, si ad alium virum per quodvis modum non fuerit sociata: post eius vero ovitum perficientur omnia, sicut superius legitur. Petrus autem famulus Sociata: post eius vero ovitum perficientur omnia, sicut superius legitur. Petrus autem famulus ad suum ovitum statim maneat liberus et abso,lutus et habeat a distributoribus auri sol. 1 byt. Neapolitanum. Denique Blactu coniux domini Sergi, quae superius consensum tribuit, legat falcidium suum Urso filio suo, si sibi supervixerit: si vero obierit infra etatem vel sine heredibus, reservat sibi facultatem facere de eo, quod voluerit, excepto de memorata terra praedictis bernaculis donata. Testator reservat facultatem iungendi, minuendi, vel etiam totum dispositum ebacuandi. Oui contra vero venire ipsi praesumpserit, componat auri libra una byt. Scriptum et actum per Andream curialem. Signum manus Sergii, qui pro occupatione mortis minime scrivere concurrit, sed omnia memorata scribere rogavit Signum manus Blactu Testes: Petrus filius domini Aligerni, Theodorus filius domini Bitali Comitis, Aligernus filius Ioannis": P. SANTAMARIA, Historia cit., pp. 209-211, doc. XIII.

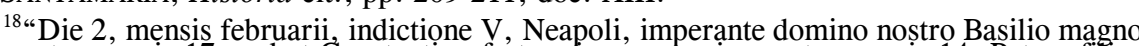
imperatore annis 17, sed et Constantino frater elus magno imperatore annis 14. Petrus filius quondam Iuliani, cui sopranomen Corbi, qui fuit habitator supra Clibum ad Sanctum Petrum ad Paternum cum consensu Eufimiae genitricis sue et Annae coniugis sue, vendit et tradit domino Stephano militi, filio quondam Leonis militis et dominae Pitru honestae feminae domino Stephano militi, tilio quondam Leonis militis et dominae Pitru honestae feminae, iugalibus, integras sexuncias suas de fundo posito in dicto loco ad Sanctum Petrum ad et ab alio latere aliis sexunciis dicti fundi, quas dictus Stephanus comparatas habebat a congregatione chartulae Sancti Petri ab illis Ferrariis distributoribus anime dicti quondam Iuliani, et ab uno capite cohaeret via, quae vadit inter ipsum fundum et fundum de illi Medici, et $a b$ alio capite est terra heredum domini Bitaliani Pellari, sicut inter se sepis exfinat. De qua etc. et a praesenti etc. et neque etc. Insuper et $a b$ omni nomine etc. propter quod accepit auri tari 14. Actamen stetit ut, quando ipse venditor ad antestandum provocatus fuerit ab emptore, ipse ostendere debeat chartulam comparationis, quam ipse genitor ipsius venditoris apprehensit a Marino Aurifice, filio quondam Ioannis Aurifici, et aliam chartulam securitatis, quam memoratus Iulianus apprehensit a Cesareo presbytero et cymiliarca Sanctae Neapolitanae Ecclesiae et a cuncta congregatione chartulae ecclesiae Stephaniae et a congregatione chartulae Ecclesiae et a cuncta congregatione chartulae ecclesiae Stephaniae et a congregatione chartulae
Sancti Petri. Poena vero in auri sol. 12 byt. Scriptum et actum per Joannem tabularium. Signum manus memorati Petri. Testes Gregorius filius domini Stephani, Theodorus filius domini Ursi et Sergius filius domini Ioannis": P. SANTAMARIA, Historia cit., pp. 211-212, doc. XIV. 
Le carte tacciono poi fino al seconda metà dell'XI secolo, quando in tre strumenti di donazione, l'uno del 16 luglio 1066, l'altro del 10 aprile 1100, l'ultimo del 25 giugno 1100, si cita, sia pure con diverse formulazioni, una "congregatio Sanctae Restitutae".

Nel primo documento, Gregorio Comitemaurone e la moglie Teodonanda, non avendo figli, offrono il ricavato di una rendita fondiaria alla "congregatio et ecclesia Sanctae Restitutae virginis et martyris intus episcopium Sanctae Neapolitanae Ecclesiae" ${ }^{19}$; nel secondo, Sergio, chiamato Leuci, offre dei possedimenti terrieri alle "congregationes sacerdotum et clericorum salutiferae carthulae congregationis ecclesiae vocabulo beatae et gloriosae Sanctae Restitutae, Christi birginis et martiris, sita vero intus episcopio iam dictae Sanctae Neapolitanae Ecclesiae"20; nel terzo, le "congregationes sacerdotum et clericorum salutiferae chartulae congregationis ecclesiae Sanctae Restitutae de intus episcopio Sanctae Neapolitanae Ecclesiae" affidano un terreno con i suoi alberi e pertinenze alla badessa del monastero di San Michele Arcangelo a Baiano ${ }^{21}$.

\footnotetext{
19“Die 16 mensis iulii, indictione IV, Neapoli. Imperante domino nostro Constantino magno imperatore annis 6. Gregorius cognomento Comitemaurone, filius domini Petri et Theodonanda, jugales, ex eo quod filios non haberent, offerunt et tradunt congregationi et ecclesie Sanctae Restitutae Christi virginis et martyra intus episcopium Sanctae Neapolitanae Ecclesiae, uncias sex, quod est medietatem cuiusdam clusuriae de terra, qui nominatur a Mearano, posita in loco, qui nominatur ad illa Conuccla, et coheret cum terra, heredis de illi, qui nominatur de Maju, et
cum terra domini Adelgisi archidiaconi Sanctae Sedis Neapolitanae Ecclesiae cum terra de illi cum terra domini Adelgisi archidiaconi Sanctae Sedis Neapolitanae Ecclesiae cum terra de illi ex eo quod dicta congregatio posuit sacras orationes quotidie, quas persolvunt sacerdotes e clerici dictae congregationis. Actum per Sergium curialem, et testes sunt Iohannes, filius Servii, et Gregorius, fillus domini Sparani": P. SANTAMARIA, Historia cit., p. 226, doc. XXVIII.

20"Die 10, mensis aprelis, indictione VIII, Neapoli. Imperante domino nostro Alexio magno imperatore annis 19, sed et Johannes Porfirogenito eius filio magno imperatore annis 8. Sergius, qui nominatur Leuci, Sanctae Neapolitanae Ecclesiae, filius quidam Leonis, habitatores de loco, qui nominatur Antinianu, iuris Sanctae Neapolitanae Ecclesiae, offert et contradit cunctas congregationes sacerdotum et clericorum salutiferae chartulae congregationis ecclesiae vocabulo beate et gloriose Sancte Restitute, Christi birginis et martire, sita vero intus episcopio iam dictae Sancte Neapolitanae Ecclesiae, idest medietatem suam de integra petia de terra, quae nominatur a Mianola, et ad illum Laccum, posita vero intus smemorato loco Antinianu, que est indivisa cum reliqua met (t) Sanctae Neapolitanae Ecclesie, et integras duo petias de terra, unam que vocatur ad illa griptula et aliam quae nominatur Ceina, positas ibidem cum omnibus etc. et ipsa medietas terre de utraque parte est secus terram de illu presbytero, qui fuit de Sançtae Neapolitanae Ecclesiae, et secus terram ecclesiam Sancti Ciriaci, et de aliis, qui ibidem adsiunt. Petia, quae nominatur ad ipse griptula, coheret sibi ab una parte terra, quae fuit de illi Barabana de Sanctae Neapolitanae Ecclesiae, quae tenere videtur a publica potestate [...]. De quibus etc. ea ratione quid ipsa congregatio illum sepellire debeat intus ipsam congregationem et omnem obsequium et officium facere quomodo iustum fuerit. Si quis autem etc. sub anathematis vinculis etc. et pena in auri sol. 100 byth. Scriptum et actum per Iohannem curialem. Signum manum memorati Leuci. Testes Cesarius filius domini Cesarii, Iohannes, filius domini Iohanni, et Iohannes, filius domini Sergii": P. SANTAMARIA, Historia cit., pp. 231-232, doc. XXXI.

21 "In nomine Dei Salyatoris nostri Jesu Christi. Imperante domino nostro Alexio magno imperatore anno nono decimo, sed et Iohanne Porphyrogenito magno imperatore eius filio anno octabo, indictione octaba, Neapoli. Certum est nos cunctas congregationes sacerdotum et clericorum salutifere chartule congregationis ecclesiae Sancte Restitute de intus episcopio Sanctae Neapolitanae Ecclesiae, a presenti die promptissima voluntate commutavimus et tradidimus vobis domna Gemma venerabilis Abbatissa monasterii beatissimi Michaelis Archageli, quod nominatur ad Bayane, Pretorio beatarum venerabilium Ancillarum Dei regionis Furcillense, una cum cuncta vestra congregatione monacharum vestri sancti et venerand monasteril, cum consensu et voluntate domni lohannis, qui dicitur Falconarii, et de domno Landulpho, uterinis germanis, filiis quondam Gregorii Falconarii, et de domno Sergio, qui nominatur Buccatorio, filio quondam domni Iohannis Buccatorii etc., idest integra petia de terra
} 
Nel corso del XII secolo, tre altri documenti, l'uno del 15 aprile 1146, l'altro del 20 giugno 1150, l'ultimo di un giorno non precisabile del 1188, riportano la medesima espressione. Nel primo, il ricco possidente Orso, detto Caballaro, figlio di Aligerno, dà mandato ai suoi esecutori testamentari (Sergio, arcidiacono della Chiesa napoletana e rettore della chiesa di San Giovanni Maggiore; Giovanni, arciprimicerio della medesima Chiesa napoletana; Sergio, chiamato Cactaldo) di provvedere alle sue ultime volontà: innanzitutto pagare i debiti ai creditori, poi, di quanto resta, donarne una parte consistente "in cunctas congregationes sacerdotum et clericorum salutiferae catholicae ecclesiae Sanctae Restitutae de intus episcopio memoratae Sanctae Ecclesiae Neapolitanae"22.

nostri iuris, pertinentes ipsius nostrae congregationis sancte ipsius ecclesie Sancte Restitute, qui nominatur Atrentula ad illum cerrum, posita in loco, qui nominatur Fullotani cum arboribus etc., de quibus nihil nobis nec a suprascripta sancta nostra congregatione exinde aliquid remanxit aut serbavimus, nec in alienam cuiuscumque persone, quod absit, commisimus aut ram commictimus potestatem etc. Et hec charta commutationis, ut supra legitur, sic firma scriptura per manum Iohannis curialis per suprascripta indictione. Petrus archiepiscopus subscripsi. per manum lohannis curialis per suprascripta indictione. Petrus archiepiscopus subscripsi. Neapolitanae Ecclesiae primicerius subscripsi. Sergius archipresbyter et cardinalis Sanctae Sedis Neapolitanae subscripsi. Petrus archidiaconus Sanctae Neapolitanae Ecclesiae subscripsi. Neapolitanae subscripsi. Petrus archidiaconus Sanctae Neapolitanae Ecclesiae subscripsi. Iohannes diaconus Sanctae Neapolitanae Ecclesiae subscripsi. Iohannes diaconus Sanctae subscripsi. Stephanus subdiaconus Sanctae Neapolitanae Ecclesiae subscripsi. Marinus subdiaconus Sanctae Neapolitanae Ecclesiae subscripsi. Ego Iohannes, filius domni Sergii testis subscripsi. Ego Iohannes curialis complevi et absolvi per suprascripta indictione": P. SANTAMARIA, Historia cit., pp. 233-234, doc. XXXIII.

22،In nomine Domini Dei Salbatoris nostri Ihesu Christi, regnante domino nostro Rocerio Siciliae et Italiae magnifico rege anno sexto decimo, et eius dominationis civitatis Neapolis anno septimo, die quinta decima mensis aprilis, indictione nona, Neapoli. Dispositu factu a me Urso, qui nominatur Caballaro, filiu quondam domini Aligerni, qui iterum Caballarum vocabatur, quaeda domina Maru iugalium personarum. De omnia mea hereditate, et domos seu sustangias de intus et de foris, quibus inferius iudicavero, firmu et stabile permaneant imperpetuum, quod dixit. Dispono primum omnium ut a meu transitu licentiam habeas quidam domino Sergio venerabili archidiacono Sancte Sedi Neapolitane Ecclesie, cognomento Guindaxio, rector et dominus de ecclesia vocabulo Sancti Iohanni Baptista Catholicae Maioris, esinde pertinet ad Sancta Neapolitana Ecclesia pro dominaticu, et domino Iohanne, venerabili archiprimicerius de ipsius Sancte Sedis Neapolitane Ecclesie, cognomento Gruccianima, et domino Sergio, cognomento Cactaldo, filiu quidam domini Sergio, qui iterum Cactaldo vocabatur, hoc est meis distributoribus, et illorum heredibus et personas illas, ad cui istut meu dispositu in manibus paruerit, appreendere et venundare, idest integris dominibus meis, positis vero intus hanc civitatem Neapolis in vico publico, qui nominatur [...], seu et integris omnes terris et fundoras et ortuas meas, que simul mihi pertinet per qualiscunque modum, que ego habere videor gradibus fabritis, que ibidem descendunt, et ipsis terris et fundoras et ortuas una cum arboribus cum palmaras et susceptorias suos, et cum omnibus intus se habentibus et pertinentibus eius, et cum introitis et antitis, seu regiis eorum, et omnibusque eis pertinentibus, ext insimul venundare illos memoratis meis distributoribus ad cuique volueris, et ad cuique esinde aliquid venundederis, firmum et stabile permaneant inperpetuum, et pretia, quod esinde tuleris, distribuas illos memoratis meis distributoribus esinde dare et pargiare debeas, idest omnes debitu meu, quod ego dare debeo ad creditoribus meis, et reliquu, qui esinde remanserint, distribuas illos memoratis meis distributoris pro anima mea in hoc ordine: in primis ad sancti mei penitentialis detur esinde aur tari quactuor, et ad quidam domino [...], venerabili abbati de monasterio ecclesie Sancti [...], uterinu germanu, dentur esinde auri tari decem, et faciant mihi esinde illa septima et omne ossequii pro me sepeliendo, et ubi tunc ipsi mei distributoris melius prebideris, dispono, ut post meu transitu siant offertu et traditu pro anima mea et de memoratis lugalibus genitoribus meis ut ic et in futuro seculo apud eternu iudice requiem de pecatis nostris imbenire baleamus apud ut ic et in futuro seculo apud eternu ludice requiem de pecatis nostris imbenire baleamus apud clericoru salutifere catholice ecclesie Sancte Restitute de intus episcopio memorate Sancte Ecclesiae Neapolitane, idest integra portione et pertinentia mea, que mihi pertinet per qualicumque modu de integra ecclesia Sancti Petri, qui ad Carbonariam, que est in pedem de 
Nel secondo documento, Giovanni Malafronte, chierico della Chiesa napoletana e figlio di Giuliano, dona un terreno, con alberi, beni e pertinenze alle "congregationes sacerdotum et clericorum salutiferae catholicae ecclesiae Sanctae Restitutae de intus episcopio Sanctae Neapolitanae Ecclesiae"23.

Monte, una cum omnibus rebus et substangiis atque possessionibus de ex ipsa portione mea de memorata ecclesia [... meliores, que ego abeo, et insimul illos in ipsius cunctas congregationes sint potestate faciendi esinde omnia, que volueris. Reliquos vero omnes mobilia mea mobiliu et immobiliu seseque mobentibus a parbu usque ad magnum capitulum pretiosu vel bile, pos meu transitu insimul rebertant et siant de quidam Drosu conius mea et de suis eredibus potestate faciendi esinde eo omnium, que volueris, asque omnia data occasione. Quoque dispono, ut $\mathrm{s}$ aliquod abuero in bene precio de rebus Sancte Neapolitane Ecclesie, post meu transitu sint ibidem datu et renditu. Abeant item memorate Sancte Neapolitane Ecclesie pro luminaria asque iniurie per tres vices ad unum". Seguono le formule di chiusura e le firme dei testimoni e del notaio: P. SANTAMARIA Historia, cit., pp. 240-242, doc. XL.

23 "In nomine Domini Dei Salbatoris nostri Ihesu Christi, regnante domino nostro Rocerio Siciliae et Italiae magnifiço rege anno vigesimo, et eius dominationis istius civitatis Neapolis anno undecimo, die vigesima mensis Iunı, indictione tertiadecima, eiusdem civitatis Neapoli. Certum est me Iohannes clericum Malafronte Sancte Neapolitane Ecclesie, filio quidam Iuliani, qui nominatur Inbuctia Carpinu, et quedam Sancte Licta memorate Sancte Neapolitane Ecclesie, iugalium personarum, a presenti die, promptissima voluntate commutabi et tradidi vobis, cunctas congregationes sacerdotum et clericorum salutifere catholice ecclesie Sancte Restitute de intus episcopio ipsius Sancte Neapolitane Ecclesie, idest integra petia de terra, que nominatur Adfera maiuri Pictuli, quod est in loco, qui nominatur Lanceasinu iuris memorate Sancte Neapolitane Ecclesie, una cum arboribus et fruttosas suas, et cum introitibus suis omnibus sibi pertinentibus, pertinente vero michi per comparatu et per commutatu per due omnibus sibi pertinentibus, pertinente vero michi per comparatu et per commutatu per due fermissime chartulẹ, e quibus ipsa chartula comparationis michi esinde fecerunt Gregorio, qui nominatur Manconi, et Stephano et Beneruso uterinis germanis, filiis quidam Sergii, qui iterum Manconi vocabatur, et quedam Letitia, iugalium personarum: ipsi autem cum consensu et voluntate de memorata Letitia genitrice illorum, simul Sancte Neapolitane Eccclẹsie [...], que vero ambe iste chartule mee una cum alia chartula commutationis, que in ipsa chartula commutationis reclaratur, ego vobis in presentis ille dedi, et apud vos remisi cum suis omnibus pertinentibus, ut superius legitur: ab uno latere est terra ecclesiae Stephanie, sicut in terre terminis est finis: ab alio latere est terra ecclesiae Sancti Ianuarii, ex ipso loco Lanceasinu iuris memorate Sancte Neapolitane Ecclesie, sicuti in terre terminis est finis, ab uno capite est terra ecclesie Sancti Pantaloni, qui fuit Sancte Neapolitane Ecclesie, que modo ipsa terra detinet domino Cesario Cacapice, sicuti in terre terminis est finis, ab alio capite est iterum terra memorate ecclesie Stephanie, set et terra domini Stephani presbiteri, qui nominatur Tiaraballo ipsius memorate Sancte Neapolitane Ecclesie, qualiter in terre terminis est finis [...]. Neque a ipsius memorate Sancte Neapolitane Ecclesie, qualiter in terre terminis est finis [...]. Neque a meis heredibus nec a nobis per personas summissas nullo tempore unquam vos memoratas cunctas congregationes sacerdotum et clericorum memorate ecclesie Sancte Restitute de intus
episcopio memorate Sancte Neapolitane Ecclesie aut posteri vestri, quod absit, abeatis exinde episcopio memorate Sancte Neapolitane Ecclesie aut posteri vestri, quod absit, abeatis exinde aliquando quacunque requisitione aut molestia per nullum modum, nec per summissas personas a nunc et imperpetuis temporibus. Insuper et omni tempore ego et heredes mei bobis vestrisque posteris et in ipsa sancta et venerabilis vestra congregatione ipsa, quae superius vobis commutabi, cum omnibus sibi pertinentibus, ut superius legitur, in omnibus illut antestare et difendere debeamus ab omnes omines omnique persona, concedentes vos et posteri vestri michi meisque heredibus [...], iḍest integra domus vestra iuris memorate sancte vestre congregationis, posita vero intus anc civitatem Neapolis, iuxta platea publica, que nominatur Summa Platea, posita vero intus anc civitatem Neapolis, iuxta platea publica, que nominatur Summa Platea, in eadem regione Summa Platea, et est ipsa domus nominata per hec membra: due inferiores cellarie modice cum due modice supperiores, et sunt una iusta alia, sicuti inter una et alia pariete est finis, ubi sunt regie, per quas ingredit de una in alia: set una ex ipsis modice superiore est costituta subtus ex parte superiore orrei ipsius memorate Neapolitane Ecclesie, et ipsa alia superiora est costituta subtus superiora orrei, et ipsa domus, que vobis adseri in ipsa commutatione pertinentem una cum superiora orrei et cum solareu aheru desuper ipsum orreum simul una cum aheribus et aspectibus suis et cum quanto vobis adseri in ipsa commutatione pertinere de gradis et vallatorio, que est de regia et antitu commune de ipsa, qualiter vobis adseri in ipsa commutatione et de domo, que est de memorata Sancta Neapolitana Ecclesia, et cum introitibus suis ad ipse modice inferiore, ubi ipsa platea publica, que regie sunt iussta ipsa platea et ad ipse modice superiore et orreum, et solareum, ubi ipsa platea publica, que plaminatur Summa Platea per memorate gradis et vallatoriu, et regia et antitu pltineu, que (1) comune, et que regie proprie ex ipșa domo, que vobis adseri in ipsa commutatione [... pertinente vero vobis per due fermissime chartule offertionis vestre, que in ipsa sancta congregatione fecit quidam Iohannes, qui nominatur Cuctillo, memorate Sancte Neapolitane Ecclesie, filio quidam domini Iohanni clerici, qui iteru Cuctillo denuo vocabatur memorate
Sancte Neapolitane Ecclesie. Set una ex ipse chartule offertionis aput vos remanxit, quod aliut 
Nel terzo documento, Giovanni Munda, figlio di Pietro, per la salvezza della sua anima e di quella dei suoi familiari, dona la sua casa, la sua terra e numerosi altri possedimenti alle "congregationes sacerdotum et clericorum salutiferae catholicae ecclesiae Sanctae Restitutae de intus episcopio Sanctae Neapolitanae Ecclesiae", perché ne dispongano come più loro aggrada ${ }^{24}$. A questi tre va aggiunto un contratto di enfiteusi, fortemente

continet, que a vestra reserbastis potestate, et quandoque michi meisque heredibus nocesse fuerit pro ipsum, quod superius vobis adseri in ipsa commutatione, tunc sicuti omni tempore vos et posteri vestri michi meisque posteris illa ostendere et mostrare debeatis, ubique michi vos et posteri vestri michi meisque posteris illa ostendere et mostrare debeatis, ubique michi
meisque heredibus necessum fuerit adque omni data occasione: et vos etiam mihi in presentis
dedistis et aput me remisistis una de ipse chartule offertionis cum una chartula venditionis, que dedistis et aput me remisistis una de ipse chartule offertionis cum una chartula venditionis, que ipse memorato Iohannes Cuctillo, et que ex parte de ipsu, quod superius vobis adseri in ipsa Servii, qui nominatur Inbuctia Carpinu, et quedam Eufimmla, iugalium personarum memorate Sancte Neapolitane Ecclesie, abitatoribus de memorato loco Eanceasinu memorate Sancte Neapolitane Ecclesie [...] et a parte meridie est pischina [...] de ipsius memorate Ecclesie, et in ipsa parte meridie est ipsum orreum ipsius memorate Neapolitane Ecclesie. Set et modicu de ipsa domo ipsius memorate Neapolitane Ecclesie quod de inferius detinet ipsu Tiaraballu, et ipsa domo ipsius memorate Neapolitane Ecclesie, quod de inferius detinet ipsu Tiaraballu, et de superius detinet memorati Ursi Sapiobu memorate Sancte Neapolitane Ecclesie, qualiter inte se pariete est finis, et a parte sẹptentrionis sunt aheres desuper ipsa platea publica, sicuti pariete est finis, ubi sunt de inferius ipse regie, per qua de inferius ibidem ipsum introitum ingredit, et de superius sunt fenestre, qui respiciunt super ipsa platea. Set ipsum orreum est costituto cum memorato solareu et aheru, et ex parte est copertu ad tectu. Iterum et aput me remisistis una chartula commutationis esinde continente, que est uterinis germanis memorate. Sancte Neapolitane Ecclesie esadelfis nepotibus suis, filiis quidam Urso, qui nominatur Sapiobu, et quedam Drosu Cuctilla, que fuit esadelfa germana sua, iugalium personarum ipsius memorate Sancte Neapolitane Ecclesia: ipsi autem per absolutionem et submictionem domıni Gregorii De gratia archiepiscopus iam dicte Sedis Sancte Neapolitane Ecclesie, et secum abendo abocatorem lohannes, qui nominatur Ruxo, sicut ipsa chartula continet". Seguono formule di chiusura e le documento, già pubblicato da Giuseppe SPARANO, Memorie istoriche per illustrare gli Atti della Santa Napoletana Chiesa e gli Atti della Congregazione delle Apostoliche Missioni eretta nel Suomo della Medesima, per Giuseppe Raimondi, in Napoli, MDCCLXXVIIII, pp $146-147$, è
particolarmente importante perche offre una descrizione precisa dell'area del complesso particolarmente importante perché offre una descrizione precisa dell'area del complesso congregazione di Santa Restituta possedeva una "domus" e un "horreum". Quanto all'orreum, non dispongo di elementi sufficienti per identificarlo con quello che compare, alla fine del XIII secolo nella vendita di un fondo, confinante con un "ortum congregationis Sancte Restitute de Neapoli", all'abate del monastero napoletano dei Santi Severino e Sossio: cfr. L'antico inventario delle pergamene del monastero dei SS Severino e Sossio 4 voll. a cura di Rosaria PILONE, Istituto Storico Italiano per il Medioevo, Roma, 1999, in part. I, pp.3 308-310 [70]. Per una nuova convincente ipotesi sulla sistemazione dell'area orientale della Cattedrale si veda Carlo Ebanista, L'atrio paleocristiano dell' insula episcopalis di Napoli, "Archivio Storico per le Province Napoletane", CXXIII (2005), pp. 51-92.

${ }^{24}$ “In nomine Domini Dei Salbatoris nostri Ihesu Christi, regnante domino nostro Guilielmus Sicilie et Italie magnifico rege anno vigesimo secondo, et eius dominationis istius civitatis Neapolis iteru anno vigesimo secondo, die secunda mensis [...], Neapoli. Certum est me Iohanne, qui nominatur de Munda, filium quidam Petri et de queda Geyta, que nominatur Caccioni qui nomium personarum, ego autem cum consensu et voluntate presenti die prontissima voluntate pro Domini Omnipotentis amore et pro redentione salbationis presenti die prontissima voluntate pro Domini Omnipotentis amore et pro redentione salbationis anime mee et de memoratis iugalibus genitoribus meis, ut ic et in futuro seculo requiem de peccatis imbenire baleam, offero et trado vobis cunctas congregationes sacerdotum et
clericorum salutifere catholice ecclesie Sancte Restitute de intus episcopio Sancte Neapolitane Ecclesie, idest integra domus mee, posite vero intus anc civitatem Neapolis [...] commune, que est intus amphiteatrum regione termensis: set offero et trado vobis [...], idest integra petia mea de terra, que est per censura quarte septem censurate a passu ferreo memorate Sancte Neapolitane Ecclesie, posita vero in loco, qui vocatur Cacciottulo, et dicitur ad casali, et illa memorata domu una cum inferioribus suis vel omnibus membris, et memorata terra cum arboribus et fruttosas suas et cum introitibus suis et omnibus generaliter et in integro pertinentibus, et coeret vero memorata integra domus, que superius vobis offeruit et tradidit portinentibus, en omnibus eius pertinentibus, ut superius legitur [...], deinceps sic offerta et tradita in vestra vestrisque posteris sint potestate, queque exinde facere volueritis, ab odierna die in vestra vestrisque posteris sint potestate, queque exinde facere volueritis, ab odierna die autem cum voluntate de memorata Palma posteriora conius mea, ut superius legitur [...], 
lacunoso, del 14 settembre 1212, nel quale pure ricorre la medesima espressione $^{25}$.

Per quanto è ad oggi nelle nostre conoscenze, il termine "Capitulum" compare per la prima volta nel 1167, in una lettera di Pietro di Blois, custode del sigillo reale e maestro del re di Sicilia, Guglielmo II, in riferimento ai chierici della Cattedrale di Napoli elettori dell'arcivescovo ${ }^{26}$. Dieci anni dopo, il medesimo termine è attestato in una bolla, nella quale l'arcivescovo di Napoli Sergio III, pregato dall'abate di Cava, Benincasa, concede a lui e ai suoi confratelli "in perpetuum", con il consenso di tutto il Capitolo cattedrale, l'esenzione dai diritti episcopali gravanti sulle proprietà napoletane dell'abbazia ${ }^{27}$. Nel 1183, in una bolla dello stesso Sergio III, si stabilisce,

personas summissas nullo tempore numquam vos memorate sanctas congregationes sacerdotum et clericorum salutifere catholice ecclesie Sancte Restitute de intus episcopio memorate Sancte Neapolitane Ecclesie, vel posteris vestris abeatis esinde aliquando quacumque requisitione aut molestia per nullum modum nec per summissas personas a nunc et in perpetuis temporibus [... et in cartula, ut superius legitur, sit firma scripta per manus Marinus curialis per memorata et in cartula, ut supperius legitur, sit firma scriptạ per manus Marinus curialis per memorata indictione. Hoc signum manus memorati loannis de Munda, ipse autem cum voluntate de memorata conius sua. Ego Martinus curialis testis subscripsi. Ego Gregorius scrittor testis subscripsi. Ego Marinus curialis complevi et
RIA, Historia cit., pp. 251-252, doc. XLIV.

25 “In nomine Domini Dei Salvatoris nostri Ihesu Christi, imperante Domino nostro Oto IV Romanorum magno imperatore, et semper augusto, anno IV, et eius dominatione civitatis Neapolis anno II, die decima quarta mensis septembris, indictione I, Neapoli. Certum est nos cunctas congregationes sacerdotum et clericorum salutifere catolice ecclesie Sancte Restitute cunctas congregationes sacerdotum et clericorum salutifere catolice ecclesie Sancte Restitute
deintus episcopio Sancte Neapolitane Ecclesie [...] a presenti die [...] propter quod vs deintus episcopio Sancte Neapolitane Ecclesie [...] a presenti die [...] propter quod vs [...]
videlicet Matheo et Mathea oc est iugales, filio et nuru quidam domini Guaimari dudu [.... et quedam domina Maria iugaliu personaru, et filia seu genero quidam domini [...], clerico de Liberto vobis odie promisimus cartula offertionis fecistis de integra domu, et de integra petia de terra in capite de ea coniunta [...] insimul posita vero intus anc civitatem Neapolim iusta bico publico, qui nominatur frigido [...] aspectibus et fructuosas suas, et super regie illorum et sulareo [..] suis, et omnibus sibi pertinentibus [..] offertionis continet, que ipsa cartula offertionis vestre nos vobis dedimus et aput vos remisimus promictimus et firmamus yobis memoratis iugalibus, ut superius legitur, ut vobis cunctis diebus vite vestre ipsa integra vobis et iam dicta orticellu. [... offeruistis, quod vos illa tenere et dominare et ibide abitamu et iam dicta orticellu. [...]. offeruistis, quod vos illa tenere et dominare et ibide abitare et recidere et frugiare debeatis et de ipsa frugias faciendi, que volueritis [...] per nullu modu tantummodo vos cunctis diebus vite vestre per omni anno [...] ad Sancta Restituta dare et [...] una $[. .$.$] iterumque promictimus vobis ut nos [...] et aiutare [...] iustu fuerit et etiam si nos vobis$ taliter facere voluerimus clarare [...]": P. SANTAMARIA Historia, cit., p. 254, doc. XLVI.

${ }^{26}$ Patrologiae Latinae Cursus Completus, Tomus CCVII, Petrus Blesensis Barthoniensis in Anglia Archidiaconus apud J. P. Migne editorem, Lutetiae Parisiorum, 1855, coll. 386-391 (Epistola CXXXI, ad E. priorem de Monasteriolo), in part. col. 390. Il racconto che Pietro fa al nipote Ernaldo sulla sua elezione all'episcopato di Napoli è particolarmente illuminante sulla funzione che svolse il Capitolo in quella circostanza: "Tu vero frequenter et ipsius papae, qu nunc sedẹt ac plerorumque cardinalium eius, qui in diebus meis legatione functi sunt, fratris etiam mei et abbatis $S$. Dionysii aliorumque magnatum, qui in terra sunt, relatione conoscere potuisti, quid cum in Sicilia essem sigillarius et doctor regis Guillelmi Secundi, tunc pueri atque post reginam et Panormitanum electum, dispositio Regni satis ad meum penderet arbitrium quidam mei aemuli machinantes me a familiaritate regis excludere, procuraverunt ut Ecclesia Neapolitana me in archiepiscopum eligeret ac per maiores Capituli ordinationem meam communi docreto et voto unanimi postularet Vocatus autem non ivi rogatus et tractus multipliciter non consensi. in prima persona dell'elezione dell'Arcivescovo si ha a Napoli nel 1318, quando, dopo la morte di Humbert d'Ormont, il logoteta del Regno Roberto di Capua, a nome di Carlo, vicario di re Roberto, si rivolse al Capitolo e lo esortò a scegliere la persona giusta per questo compito, grata a Dio e agli uomini, o almeno regnicolo: Domenico AMBRASI, La vita religiosa, in Storia di Napoli. III. Napoli angioina, Società Editrice Storia di Napoli, Napoli, s.d.[1969], pp. 437573 , in part. p. 452 .

27 “Sergius Dei gratia Neapolitanus archiepiscopus, dilecto in Christo Benincasae eadem gratia Cavensi abbati et fratribus eiusdem monasterii eorumque successoribus, in perpetuum Ad pontificatus nostri honorem et sollicitudinem non propriis meritis, sed solius divinae gratiae 
"una cum Capitulo Ecclesiae nostrae", che chiunque dei chierici della Chiesa di Napoli muoia tra le calende di marzo e quelle di novembre può godere della facoltà di decidere liberamente a chi destinare i proventi del proprio beneficio, mentre chi muoia tra le calende di novembre e quelle di marzo ha la facoltà di destinare a chi voglia la metà dei medesimi proventi ${ }^{28}$.

munificentia evocati, tanto movemur sacris et venerabilibus locis diligentiori pietatis et caritatis intuitu providere, religiosorumque virorum in his quae secundum Deum possumus et debemus, assensum iustis precibus et postulationibus adhibere quanto ipsi a mundi huius solicitudinis segregati, cura et studio cautiori in locis eisdem Dei omnipotentis laudibus et obsequiis dignoscuntur insistere, et propensius et solicitius pro omnium animarum utilitate et aeterna salute invigilare. Ea propter, carissime et dilecte plurimum in Christo frater Benincasa, prefat monasterii venerabilis abbas, divini amoris contemplatione inducti, et tam ob laudabbilem eiusdem monasterii, cui Deo auctore preestis, quam vestram et fratrum vestrorum religionem et honestatem, vestris dignis petitionibus aures duximus inclinandas. Communi ergo consens ac voluntate fratrum nostrorum diaconorum, cardinalium, subdiaconorum, totiusque Capituli nostri, concedimus vobis et successoribus vestris in ecclesiis Sancti Arcangeli, quae posita est sub muro civitatis foris porta de illis monachis, et Sancti Gregorii de Legionario monasterii videlicet vestri cum suis ecclesiis, et Sanctae Mariae, quae constructa est in regione Portanovensi, iuxta domum de illis Accapacciis, aliisque omnibus ecclesiis vobis pertinentibus, quas Cavense monasterium in Civitate Neapolitana habere dignoscitur, omnem libertatem, quas Cavense monasterium in Civitate Neapolitana habere dignoscitur, omnem libertatem, quatenus absque omni contradictione nostra nostrorumque successorum, seu qualibet exactione quatenus absque omni contradictione nostra nostrorumque successorum, seu qualibet exactione omnia, quae concessione pontificum, liberalitate regum, ducum, principum, comitum et baronum, vel oblatione quorumlibet fídelium nunc in praesentiarum possident aut in futurum poterunt adibisci, libere et absolute possidere. Praetaxata etiam divinae caritatis pietate et liberalitate, concedimus ipsis prefatis ecclesiis, ut chrisma, oleum sanctum, consecrationes ipsarum ecclesiarum suorumque altarium, ordinationes monachorum vel clericorum, quando in ipsis ecclesiis deservire videbuntur, a nobis et a nostris successoribus, si gratis et absque gravitate dare voluerint, recipiatis: alioquin liceat vobis a quocumque catholico episcopo haec omnia libere et absolute recidere. Concedimus quoque praedictae ecclesiae Sancti Archangeli parochiam, quae continetur in instrumento diffinitionis, quae diffinitio facta est inter ecclesiam pancti Iohanni A postoli et Evangelistae, quae nominatur in Curte, et praefatam ecclesiam Sancti Archangeli tempore bonae memoriae abbatis Marini, baptisterium, coemeterium et processiones; ut, quicumque in eadem ecclesiam sibi sepolturam eligerint, libere eos recipere possitis Et quicumque monachi vel clerici in ipsis ecclesiis morabuntur per nos sive per nostros successores ac per nostrum Capitulum minime constringatur. Si quis autem huius nostri privilegii temerator aut praevaricator exstiterit, et huic nostrae concessioni contraire praesumpserit, excommunicationis vinculo innodatus inferni tormenta horribilia sortiatur. Qui vero, quod concedimus et statuimus, observaverit, orationum et beneficiorum omnium, quae in praenominato monasterio fiunt, particeps fiat, et aeternae beatitudinis gaudiis et requie perfuatur, Amen. Huius autem concessionis seriem scribere mandavimus Nicolao notario nostro

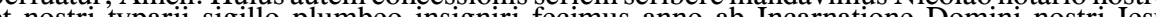
et nostri typarii sigillo plumbeo insigniri fecimus anno ab Incarnatione Domini nostrị lesu Christi millesimo centesimo septuagesimo septimo, mense mail, indictione decima, nostri vero archiepiscopatus anno secondo. Ego Sergius Neapolitanus archiepiscopus subscripsi., Ego Iohannes archipresbiter et cimiliarcha Sanctae Neapolitanae Ecclesiae subscripsi”: SANTAMARIA Historia, cit., pp. 247-248,
CHIOCCARELLO, Antistitum cit., p. 134.

28، In nomine Dei aeterni et Salvatoris nostri Iesu Christi. Regnante domino nostro Guilielmo Dei gratia Siciliae et Italiae magnifico rege, anno Dominicae Incarnationis millesimo centesimo actuagesimo tertio, die vicesima secunda mensis madii, primae indictionis. Nos Sergius Dei clericorum tam nostrae maioris ecclesiae, quam totius parochiae ipsius propriae, pro parvitate beneficiorum in suprema egestate vitam finire, una cum Capitulo Ecclesiae nostrae, pro salute animarum praedecessorum nostrorum et nostra, nostrorumque successorum, a presenti die stabilimus et praesentis paginae perpetua firmitudine roboramus, et tam in nostra Maior Ecclesia Neapolitana, quam in caeteris ecclesiis propriae parochiae nostrae civitatis, inviolabili censemus sanctione perpetua observandum ut, quicumque clericorum tam nostrae maioris ecclesiae quam totius propriae parochiae ipsius, humanae naturae debitum soluturus a kal. martii usque ad kal. novembris de hac luce migraverit, liberam habeat facultatem indicandi et martil usque ad kal. novembris de hac luce migraverit, liberam habeat facultatem indicandi et dimittendi, ubi et cui voluerit totum proventum beneficii sui, quod ante sive in civitate, sive tempore, et tam de Maiori nostra Ecclesia quam de aliis ecclesiis et etiam congregationibus, sive in terris, sive in domibus, et in quibuscumque aliis, iuste aliquid aliquo modo usque ad kal. 
In un documento del gennaio del 1213, l'arcivescovo di Napoli, Anselmo, si rivolge ad Egidio, cimiliarca e chierico della "congregatio Salvatoris", in risposta ad una supplica che lo stesso Egidio, insieme con i suoi confratelli della congregazione del Salvatore, gli aveva rivolto, in nome dei servizi che continuamente e devotamente essi prestavano alla Chiesa napoletana, lamentandosi dello stato di povertà in cui versavano a causa dei pagamenti delle collette. L'arcivescovo, sollecitato dal Capitolo che era intervenuto in difesa della suddetta congregazione, aveva deciso di accogliere le richieste. Pertanto, ai membri della congregazione del Salvatore faceva la speciale grazia di esentarli da ogni pagamento, tranne in determinati casi ${ }^{29}$.

novembris fuerat habiturus: et quicumque a kal. novembris usque ad kal. martii moriturus vitam finiverit, liberam habeat similiter potestatem indicandi et dimittendi, cui vult et ubi vult, medietatem proventus illius anni sequentis totius beneficii sui, tam de ecclesiis quam de congregationibus er de aliis omnibus, sicut praedictum est, et aliam medietatem habeat, quicumque sibi canonice successor exstiterit: a fịne vero anni defunti omnia in usum successoris cedant. Praetera protestatione fermissima statuimus et inrevocabili munimine prohibemus, ut de proventu beneficii cuiuscumque nemini liceat pro quarta parte seu pro quarta, ut vulgo dicitur, nec sub obtentu aliquo dominii vel consuetudinis civitatis, aliquid accipere. Si quis autem pertinax aut indevotus hanc nostrae institutionis et confirmationis paginam, sive in parte, autem pertinax aut indevotus hanc nostrae institutionis et confirmationis paginam, sive in parte, Dei Omnipotentis et beatae Mariae semper Virginis, beatorum apostolorum Petri et Pauli, et beati Ianuarii et Agrippini, quorum patrocinio communimur, et aliorum sanctorum, quorum reliquie in praedicta nostra ecclesia continetur, et nostra, anatema maranatha, et cum Iuda proditore et Nerone perennem habeat portionem. Qui autem huiusmodi observator exstiterit, divinae benedictionis presidio potiatur, et aeternae beatitudinis praemia inter sanctorum collegia consequatur. Ut autem firmius habeatur, signo confirmavimus et sigillo nostro fecimus insigniri. Facta Neapoli anno, die et indictione praetaxatis, perpetuo valitura. Hoc adhuc ex communi consilio et voluntate duximus inserendum, et perenni robore confirmandum ut, si quis

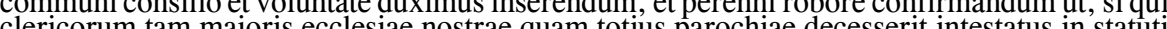
clericorum tam maioris ecclesiae nostrae quam totius parochiae decesserit intestatus in ștatutis temporibus, ut supra dicitur, de preventu beneficii sui, sicut est superius ordinatum, si super eum manifestum et certum debitum apparuerit, prius solvatur; reliquum denique, si quid est residuum partium defunti intestati, cum uno vel duobus de proprinquioribus suis, qui sibi in vita sua plus placuerint, per notitiam Capituli nostrae Ecclesiae, pro anima eius distribuant. Si quis autem contra hoc sciens venerit, nisi monitus resipisçat, praedicto sit anathemate innodatus. Hoc quoque sub dicto anno, die et indictione additum sit perpetuo munimine confirmatum atque approbatum. Sergius archiepiscopus subscripsi. Ego loannes archipresbyter et cimeliarcha Sanctae Sedis Neapolitanae Ecclesiae subscripsi. Ego Guilielmus presbyter cardinalis Sanctae Sedis Neapolitanae Ecclesiae subscripsi. Ego Ioannes presbyter cardinalis Sanctae Sedis Neapolitanae Ecclesiae subscripsi. Ego Ioannes presbyter cardinalis Sanctae Sedis Neapolitanae Ecclesiae subscripsi. Ego Marinus Neapolitanae Ecclesiae diaconus subscripsi. Ego Matthaeus Neapolitanae Ecclesiae diaconus subscripsi. E o Sergius Neapolitanae Ecclesiae diaconus subscripsi. Ego Riccardus Neapolitanae Ecclesiae diaconus subscripsi. Ego Ioannes Nubscripsi. Ego Riccardus Neapolitanae Ecclesiae diaconus subscripsi. Ego loannes Nubdiaconus subscripsi. Ego Ioannes Neapolitanae Ecclesiae subdiaconus subscripsi. Ego subdiaconus subscripsi. Ego Ioannes Neapolitanae Ecclesiae subdiaconus subscripsi. Ego subdiaconus subscripsi. Ego Sergius Neapolitanae Ecclesiae subdiaconus subscripsi. Ego Donadeus Neapolitanae Ecclesiae subdiaconus subscripsi": P. SANTAMARIA Historia, cit., pp. 249-251, doc. XLIII.

29،Anselmus miseratione divina Neapolitanus archiepiscopus dilectis in Domino filiis Aegidio cimiliarchae et clericis congregationis Salvatoris in perpetuum. Piae postulatio voluntatis effectu debet prosequente compleri, quatenus et devotione sinceritas et utilitas postulata vires indubitanter assumat. Cum ergo, dilecte et carissime in Domino filii, Aegidii, cimeliarcha Neapolitanae Ecclesiae, tu et socii tui clerici congregationis Salvatoris humiliter et frequenter supplicaveritis nobis, ut intuitu obsequiorum, quae iugiter ac studiosius impendebatis Ecclesiae Neapolitanae circa celebrationem divinorum officiorum, providere deberemus pauperi congregationis vestrae Salvatoris, videlicet super gravaminibus et damnis, quae occasione collectarum substinuerat, et frequentius substinebat; nos tam ad supplicem postulationem vestram, quam et ad preces Capituli pro vobis nobis attente porrectas, supplicationes vestras tandem de ipsius Capituli consilio benigne super praemissis duximus admittendas. Unde nos, volentes vobis et posteris vestris, qui divinis officils et ecclesiasticis obsequils Neapolitanae Ecclesiae iugiter insudatis, super hoc gratiam facere specialem auctoritate praesentium dictam congregationem vestram Salvatoris ab omni collecta eximimus 
Nel 1255 il papa Alessandro IV, ricordando la concessione di Sergio III, conferma all'arcivescovo di Napoli, al Capitolo e a tutto il clero della città e della diocesi di Napoli, che qualsiasi Canonico o chierico della Cattedrale o delle altre chiese cittadine e diocesane muoia tra le calende di marzo e quelle di novembre, abbia il diritto di lasciare a chiunque voglia i proventi del suo beneficio, mentre chi muoia tra le calende di novembre e quelle di marzo ne può lasciare la metà, essendo l'altra metà riservata a colui gli succede ${ }^{30}$.

Quasi un secolo dopo, mentre la nuova cattedrale era già in via di completamento, il 2 settembre del 1309 Bartolomeo Siginolfo, Conte di Caserta e Gran Camerario del Regno di Sicilia, donava i suoi beni al primicerio, ai diaconi, ai cardinali e agli altri Canonici della Chiesa napoletana, "congregationem facientes in ecclesia Sanctae Restitutae de Neapoli", a lode dell'Altissimo Creatore, della Vergine Madre di Dio e di Santa Restituta vergine e martire, "sub cuius vocabulo ecclesia vestrae congregationis, quae vocatur Sancta Restituta, laudabiliter insignitur" ${ }^{\prime 3}$.

et amodo censemus immunem, statuentes ut et vos fratres dictae congregationis Salvatoris tunc solum collectis faciendis teneamini conferre, quando et alii clerici tam nostrae Maioris Ecclesiae, quam et omnes alii de civitate ad contributum collectarum, quae inciderint, personaliter vocabuntur": P. SANTAMARIA Historia, cit., pp. 255-256, doc. XLVII.

30“Alexander episcopus servus servorum Dei venerabili fratri archiepiscopo et dilectis filiis, Capitulo et clero civitatis et diocesis Neapolitanae, salutem et Apostolicam benedictionem. Provenit ex devotione nostrae mentis, ut petitionibus vestris favorabiliter animadvertentes gratiam, quam praesertim suadet honestas, vobis liberaliter faciamus. Sane, prout a vobis ex parte vestra petitio continebat, bonae memoriae Neapolitanus archiepiscopus pradecessor tuus, frater archiepiscope, de consensu Capituli, fecit statutum, ut unusquisque Canonicus vel clericus maioris et aliarum ecclesiarum civitatis et dioecesis Neapolitanae a kal. martii et infra usque ad kal. novembris diem extremum claudens beneficiorum suorum dictarum ecclesiarum
civitatis et dioecesis proventus, quos erat ab eisdem kal. martii usque ad praefatas novembris civitatis et dioecesis proventus, quos erat ab eisdem kal. martii usque ad praefatas novembris
kal. ex ipsis beneficiis proventus percepturus, posset indicare et dimettere, cui vellet: et hoc kal. ex ipsis beneficils proventus percepturus, posset indicare et dimettere, cui vellet: et hoc ad kal. martii rebus eximeretur humanis, et successor eius haberet reliquia proventuum. Idem praeterea inhubuit pradecessor, ne quis sub quovis obtentu dominii ab aliquo praedictorum ratione quartae partis proventuum beneficiorum, quae quarta vulgariter dicitur, quicquam praesumeret accipere, vel etiam extorquere, prout in instrumento publico inde confecto plenius dicitur contineri. Nos igitur, quod in hac parte provide factum est, ratum habentes et gratum, id vestris supplicationibus inclinati auctoritate Apostolica confirmamus, et praesentis scripti patrocinio communimus. Nulli ergo omnino hominum liceat hanc paginam nostrae confrimatiopatrocinio communimus. Nulli ergo omnino hominum liceat hanc paginam nostrae confrimationis infringere, vel ei ausu temerario contraire; si quis autem hoc praeseumpserit, indignationem Neapoli, X kal. iunii, pontificatus nostri anno primo": P. SANTAMARIA Historia, cit., pp. 260261, doc. L.

31"Die 2 septembris 1309 Bartholomeus Siginulfus de Neapoli, comes Casertae, magnus Regni Siciliae camerarius, venerabilibus viris dominis primicerio diaconibus, cardinalibus, et Regni Siciliae camerarius, venerabilibus viris dominis primicerio, diaconibus, cardinalibus, et
caeteris aliis Canonicis Ecclesiae Neapolitanae congregationem facientibus in ecclesia Sanctae caeteris aliis Canonicis Ecclesiae Neapolitanae congregationem facientibus in ecclesia Sanctae Quoniam, ut ait Apostolus, omnes stabimus ante tribunal Christi, recepturi, prout in corpore egimus, sive bonum fuerit, sive malum, oportet nos diem rationis extremae misericordiae operibus prevenire [...]. Ideo huiusmodi considerationis intuitu ob remissionem peccatorum tam propriorum, quam parentum nostrorum, ex mera nostra liberalitate ad laudem et reverentiam Altissimi Creatoris, beatae Mariae Virginis Matris Eius, beatae Restitutae virginis et martyris, sub cuius vocabulo ecclesia vestrae congregationis, quae vocatur Sancta Restituta laudabilite insignitur , ac omnium sanctorum, castrum novum Sancti Angeli Montis ad Cristam cum

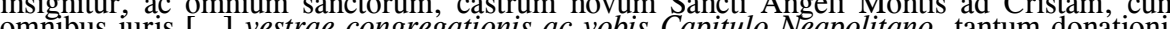
omnibus iuris [...] vestrae congregationis ac vobis Capitulo Neapolitano, tantum donationis titulo donamus, concedimus, et transferimus irrevocabiliter, quoad proprietatem et possessionem ad habendum, tenendum, possidendum, per nostrum anulum, nomine vestro et dictae vestrae congregationis, venerabilem dominum Guilelmum de Tocco, diaconum et canonicum vestrum exinde investientes [...]: P. SANTAMARIA, Historia cit., pp. 266-267, doc. LIV. 
L'11 settembre del 1317, quando il nuovo edificio cattedrale doveva esser stato ormai completato, l'arcivescovo provenzale Humbert d'Ormont emanava una bolla "de divinis officis" nella quale stabiliva i compiti del Capitolo cattedrale "ad divinum cultum augendum" 32 . Nel testo, redatto e reso pubblico con il consenso del medesimo Capitolo per porre un freno alle negligenze di alcuni dei suoi membri, si fa riferimento esclusivamente ai Canonici e non si menzionano altre congregazioni ${ }^{33}$.

In un documento del 17 gennaio 1332 i Canonici della Cattedrale e tutta la congregazione di Santa Restituta si impegnano a celebrare due anniversari all'anno "in perpetuum", uno dei quali nel giorno festivo di santa Caterina, per l'anima di un certo Giovanni Costantino, il secondo nella festa dei santi Erasmo, Marcellino e Pietro, per l'anima di donna Isabella, moglie

\footnotetext{
32 "Nos Umbertus miseratione divina Neapolitanus archiepiscopus omnibus volumus esse notum, quod suscepti regiminis nos cura solicitat, ut utilitatibus subiectorum, praecipue clericorum nostrorum, in illis maxime, per quae animarum saluti consulitur, bonorum statutorum editione solicite providere curemus. Sane cum desideremus divinum cultum augeri, non minui, et nonnulla de nostro Capitulo Neapolitanae Ecclesiae multum negligenter conveniant ad divina diebus et horis debitis, quibus ad suprascripta tenentur. Idcirco de consensu et voluntate dicti nostri Capituli infrascriptis constitutionisbus perpetuo valituris diximus statuendum: I. Quod omnes clerici nostrae Maioris Ecclesiae Neapolitanae, quocumque nomine censetur, diebus et horis debitis, quando intrare et interesse debent divinis officiis, ad ipsa conveniant, ut tenentur; ut Deo deinde Ecclesiae satisfaciant, et divinam nedum nostram ipsa conveniant, ut tenentur; ut Deo deinde Ecclesiae satisfaciant, et divinam nedum nostram mereantur effugere ultionem. II. Item statuimus ut quotiens officium mortuorum pro celebratur, omnes solicitae conveniant ad dictum officium celebrandum; et si quis adeo negligens fuerit, quod post introitum misssae ad dictum officium convenerit, et nọn ante portione quam habere debet interessendo a principio dicti officii, sit ea vice ipso facto privatus. III. Item statuimus quod, quotiens festum aliquod celebratur in nostra Maiori Neapolitana Ecclesia, in quo pecunia aliqua pro prandio vel pro aliquo alio inter clericos dividatur, si quis Canonicorum sic negligens fuerit, quod in vesperis ad primum Gloria Patris post primum psalmum, ad matutinum ad primum nocturnum psalmorum et in missa ad epistolam completam non convenerit ad divina officia, non obstante quod post ad officia ipsa convenerit, portione de dicta pecunia contingente ipso facto ea vice privetur. IV. Item statuimus quod, quotiens divina officia in nostra Maiori vel Sancte Restitutae Ecclesiis solemniter celebrantur, nullus clericorum, quocumque nomine aut dignitate refulgeat, chorum intrare audeat, nisi superpelliceo et almucia
sit indutus, nec in choro, sed extra se induat et sic indutus chorum ipsum intret ad ipsa divina Sit indutus, nec in choro, sed extra se induat et sic indutus chorum ipsum intret ad ipsa divina
officia celebrandum. Qui vero contrarium fecerit, si aliquid sit inter ipsos clericos dividendum, per nos postea poena debita iuxta nostrum arbitrium puniatur. Mandamus tenere haeec statuta cellarariis et illis, qui dividere habent inter ipsos pecuniam, sub poena excommunicationis, quam eos, si contrarium fecerint, incorrere volumus ipso facto ut nulli venienti contra statuta praedicta aliquid deferant in hac parte. Datum Neapoli in nostro Archiepiscopali Palatio anno Domini MCCCXVII, die XI mensis septembris, I indictione, pontificatus domini Iohannis Papae XXII anno primo": Alessio Simmaco MAZZOCCHI, Dissertatio historica de Cathedralis Ecclesiae Neapolitanae semper unicae variis diverso tempore vicibus Novello de Bonis Typographo Archiepiscop. et Iosepho eius filio, Neapoli, M̈BCCLI, pp. 149 SS.

${ }^{33}$ Cosimo Damiano FONSECA, Vescovi, capitoli cattedrali e canoniche regolari (sec. XIV$X V I$ ) in Vescovi e diocesi in Italia dal XIV alla metà del XVI secolo. Atti del VII Conveono di storia della Chiesa in Italia (Brescia, 21-25 settembre 1987), 2 voll., a cura di Giuseppina De Sandre Gasparini, Antonio Rigon, Francesco Trolese, Gian Maria Varanini, Herder, Roma, 1990 I pp. 83-105, in part. p 91, nell'analizzare i rapporti tra arcivescovo e Capitolo a seguito della costruzione della nuova cattedrale di Napoli, ha fatto osservare che le motivazioni della Costituzione di d'Ormont "esaltano le prerogative canoniche del vescovo, l'espletamento di compiti istituzionali di governo, più che il rilevante ruolo del capitolo nell'ambito organizzativo della Chiesa locale".
} 
di Giovanni, in cambio di una ricca proprietà terriera donata ai Canonici da Golino Costantino, figlio dei due beneficiari delle messe ${ }^{34}$.

34، In nomine Domini Dei et Salvatoris nostri Iesu Christi. Anno ab Incarnatione eius millesimo trecentesimo trigesimo secondo, regnante domino nostro Roberto Herusalem et Siciliae, Ducatus Apuliae et Principatus Capuae inclyto rege, Provinciae et Forcalcherii ac Pedimontis comite, regnorum eius anno vigesimo quarto, die decima septima mensis Ianuarii, indictione quintadecima, Neapoli. Certum est nos Gregorio clerico cognomine Marogano, filio quondam domini Macarii Marogani et quondam dominae (deest nomen) honeste femine iugalium personarum, et Bartholomeo umili presbitero cognomine Scotto, filio domini Corradi Scotto et quondam domine Marine, honeste femine, iugalium personarum, insimul Canonici Maioris Ecclesie Neapolitane et cellararii seu procuratores de cuncta congregatione sacerdotum ecclesie Sancte Restitute deintus episcopio: nos autem tam pro parte nostra quam pro parte vice et nomine de ipsa cuncta congregatione sacerdotum et clericorum supradicte ecclesie Sancte promettimus tibi domino Golino umili presbitero Constantino, filio quondam magistri loannis promettimus tibi domino Golno umili presbitero Constantino, filio quondam magistri loannis per convenientiam tu nobis pro parte nostra et pro parte vice et nomine ipsius congregationis seu et per nos in ipsa ecclesia. Sancte Restitute, offeruisti et tradidisti irrevocabiliter per firmissimam chartulam offertionis et traditionis, quam tu nobis feristi et in ipsa congregatione et in praedicta ecclesia Sancte Restitute, quam penes non habemus, idest illa integra petia de terra, quae fuit tua, quae modo est de ipsa congregatione, et quae predicta petia de terra est per mensura medium unum et quartae partis censurata ad paxum ferreum praedictae Sancte Neapolitane Ecclesiee, posita vero dicta petia de terra in loco qui nominatur Calvictiano et dicitur de Sacca-picta, insimul cum arboribus et fructibus suis et cum aliis omnibus habitis et pertinentibus, et cum introitu et exitu suo, et cum omnibus sibi pertinentibus, et quae praedicta petia de terra est coniuncta cum terra monasterii Sancti Severini, unde per exinde ad ipsam petiam de terra quam tu nobis et per nos in ipsa congregatione et ecclesia offeruisti et tradidisti petiam de terra quam tu nobis et per nos in ipsa congregatione et ecclesia offeruisti et tradidisti, introitum ingredit, et cum terris ecclesie Sancti Petri de illis Ferrariss, et cum terra Sanctorum Cosme et Damiani; proinde et nos suprascripto Gregorio clerico cognomine Marogano et praedicto Bartholomeo cognomine Scotto umili presbitero insimul cellararii seu procuratores episcopio, nos autem tam pro parte nostra quam pro parte vice et nomine de ipsa congregatione Restitute, ut super legitur, per ipsa convenientia et per hanc chartam seu et per pactum et conventionem quas nos tibi fecimus, quando tu nobis et ad suprascriptam congregationem se et ad ipsa ecclesia Sancte Restitute offeruisti et tradidisti suprascripta petia de terra quae fuit tua tua Golino umili presbitero Constantino, ut super legitur, quatenus tenuti et obligati siamus nos et posteri seu subcexores nostri, et ipsa congregatio seu suprascripta ecclesia Sancte Restitute posteri seu subcexores nostri, et ipsa congregatio seu suprascripta ecclesia Sancte Restitute, facere de nostro proprio, seu de proprio de ipsa congregatione per omnia annuo usque in perpetuum idest duo anniversaria, unumquemque de tarenis tribus in carolenis de argento giliatis boni et iusti ponderi ana due carotenis de argento pro tari computatis, videlicet unum novembris pro anima suprascripti quondam magistri Ioannis Constantini olim patris tui, et alium anniversarium facere debeamus per omnia annuo in festo sanctorum Eraxmi et Marcellini et Petri de mense iunii pro anima suprascripte quondam domine Isabelle, honeste femine, olim matris tue, asque omni pigritia usque in sempiternum. Et ad maiorem cautelam tuam et predictarum animarum de sumrascriptis iugalibus genitoribus tuis, nos tibi tuisque heredibus seu prescexoribus pro exinde de presenti specialiter et expresse obligamus suprascriptam petiam de terra, quae fuit tua que modo est de suprascripta congregatione, et alia omnia bona de ipsa terra, quae fuit tua, que modo est de suprascripta congregatione, et alia omnia bona de ipsa congregatione mobilia et stabilia ad ipsa congregatione intus pertinentia de omnia suprascripta inviolabiliter actendere et observare, ad penam unciarum sex, medietate ipsius pene componenda tibi tuisque heredibus seu subcexoribus, et alia medietatem dicte pene ego
suprascriptus Petrus de Gaudioso curialis, tamquam persona publica pro parte predicte Curie sollemniter stipulatus sum a predictis cellarariis seu procuratores, et reliqua medietate dicte pene tu supradicto domino Golino pro parte tua et heredum seu subcexorum quorum sollemniter nobis predictis cellarariis seu procuratoribus stipulatus fuisti. Et soluta suprascripta pena, vel non soluta, si committi contigerit, nihilominus presens charta sive contractus cum iis, que continet, in robore firmatis perseveret, et haec carthula, ut super legitur, sit firma scripta per manus suprascripti Petri de Gaudioso curialis per suprascripta indictione". Seguono le firme del chierico Gregorio Marogano e del presbitero Bartolomeo Scotto, cellararii della congregazione di Santa Restituta, poi quelle del primicerio, del cimiliarca, di tre cardinali, di sedici Canonici della Cattedrale (uno dei quali "decretorum doctor"), di un canonico capuano, di quattro diaconi, e il segno della croce di un presbitero "nescientis scribere": P" di quattro diaconi, e il segno della croce di un
SANTAMARIA, Historia cit., pp. 269-273, doc. LVI. 
Pochi anni dopo, il 20 ottobre del 1334, l'arcivescovo Giovanni Orsini emanava una bolla "de officiis funerariis", altrimenti conosciuta come Constitutio exequialis o super exequiis, al fine di disciplinare l'organizzazione e la gestione delle esequie. I ventidue punti di cui essa si compone prevedevano le direttive di comportamento da osservarsi da parte del corpo clericale facente capo alla Cattedrale: innanzitutto del "Capitulus nostrae Maioris Neapolitanae Ecclesie" e poi dei diversi mansionari che coadiuvavano i Canonici nello svolgimento degli uffici e delle processioni, dai semplici "sacerdotes et alii clerici" al "magister scholarum Maioris Neapolitanae Ecclesiae".

Attenzione particolare era riservata da Orsini a tre temi: la successione gerarchica dei chierici dietro la croce, il numero delle candele da portare in processione, la decenza e l'adeguatezza delle vesti ${ }^{35}$. Come si evince dal passo introduttivo della Constitutio, l'arcivescovo Orsini agì con il pieno consenso del Capitolo ${ }^{36}$, insieme al quale sancì la validità perpetua delle regole incluse nella nuova Constitutio "In nomine Domini nostri Jesu Christi Amen. Anno Nativitate eiusdem 1334. Pontificatus Sanctissimi in Christo Patris et Domini Domini Ioannis Divina Providentia Papae XXII Anno XVIII. Nos Ioannes Dei et Apostolicae Sedis gratia archiepiscopus Neapolitanus, ad nostrum munus nos innatae charitatis instinctus et auctoritas pastoralis inducit, ut sicut vivorum actibus et decentibus ordinationibus studiosus intendamus etiam ne clerici inordinate remaneant, vigilem curam et sollicitudinem impendamus. Quapropter cum consensu nostri Capituli statuendo ordinamus atque firmamus infrascriptis ordinationes perpetuo valituras" ${ }^{37}$.

Al testo di Orsini del 1334, trascritto dal canonico Alessio Simmaco Mazzocchi alla metà del Settecento sulla base di un manoscritto seicentesco di Camillo Tutini conservato nella Biblioteca Brancacciana ${ }^{38}$, fecero seguito, solo tre anni dopo, il I di maggio del 1337, le sessantaquattro Constitutiones diocesane destinate a restare per più due secoli, fino all'emanazione delle norme sinodali post-conciliari del 1565, l'unica norma vigente del clero napoletano ${ }^{39}$. Le nuove Constitutiones di Orsini furono confermate sia

\footnotetext{
${ }^{35}$ Negli anni di governo di Orsini, erano entrati a far parte del Capitolo, per volontà del re Roberto d'Angiò numerosi membri delle più illustri famiglie del Regno. Il re in persona aveva chiesto al papa Giovanni XXII la dignità canonicale per i suoi protetti: D. AMBRASI, La vita religiosa cit.

${ }^{36} \mathrm{~A}$ tal proposito, C. D. FonsECA, Vescovi, capitoli cattedrali e canoniche regolari cit., ha precisato che nelle Costituzioni orsiniane del 1337 "vengono esaltati il nostrum munus, l'instinctus innatae charitatis l'auctoritas pastoralis, la vigilis cura, la sollecitudo dell'ufficio episcopale riducendo il Capitolo ad un ruolo di subalternita in quanto ad esso viene affidato un servitium minutomente regolomento e e ond subalternita, in quanto ad esso viene affidato un servitium minutamente regolamentato e scandito nelle sue varie articolazioni; né esalta il suo protagonismo la circostanza che sia il d'Ormont sia l'Orsini esercitano il diritto di statuizione «de consensu et voluntate dicti Capituli», trattandosi di una prassi normale e canonicamente consolidata e, quindi, di un atto dovuto".

${ }^{37}$ A.S. MAZZOCCHI, Dissertatio historica cit., p. 157.

${ }^{38}$ Il manoscritto tutiniano è probabilmente da identificarsi con il codice della Biblioteca Nazionale di Napoli, Branc. I. F. 2.

${ }^{39}$ Sulle vicende della tradizione manoscritta delle Costituzioni orsiniane: Domenico MALLARDO, La Pasqua e la Settimana Maggiore a Napoli dal secolo V al XIV, "Ephemerides Liturgicae", LXVI (1952), pp. 3-36; sulf uso posteriore: Romeo DE MAIO, Le origini del
} 
dall'arcivescovo Gaspare de Diano nel 1440 (che vi aggiunse le costituzioni numero 65 e 66, una rubrica della messa di san Gennaro ed una scomunica "contra choreantes seu convivia facientes in ecclesiis") ${ }^{40}$, sia da Alessandro Carafa nel 1484, sia infine da Francesco Carafa nel $1542^{41}$. In esse compare per la prima volta, nella prefazione dell'arcivescovo Orsini, il termine "Hebdomadarii", per definire quei chierici che avevano la mansione di sostituire i Canonici del Capitolo nella celebrazione delle messe feriali ${ }^{42}$ : "Cum in hac nostra Neapolitana Ecclesia certi ritus modi et consuetudines ab antiquo fuerint et debeant observari [...], volentes ut [...] divinu culti per quemlibet iuxta veram et probatam consuetudinem serviatur, consuetudines ipsas, quas depositionibus plurium venerabilium virorum diaconorum, cardinalium, Canonicorum, Hebdomadariorum praedictae nostrae Ecclesiae, ac aliorum nobilium militum vitae et opinionis ecclesiasticae, probatas, observatas et ostentas esse comperimus, in hoc volumine, prout capitulatim infra disseritur, duximus ad perpetuam rei memoriam redigendas" 43 .

Le dichiarazioni introduttive di Orsini chiariscono fuor di dubbio che si trattava di una collezione di consuetudini cerimoniali e di riti liturgici per gran parte già in uso nella Cattedrale di Napoli. Ciò non toglie che è qui che per la prima volta si sancisce la distinzione tra il clero maggiore (il Capitolo cattedrale) e i componenti del clero inferiore (gli Ebdomadari, i Quaranta), con una precisione terminologica nuova che quanto meno indica che si era attuata una codificazione delle gerarchie interne al clero episcopale.

Riassumendo i dati che emergono dai documenti e dalle costituzioni diocesane, si evince che, prima della costruzione della nuova cattedrale napoletana di patrocinio angioino, due documenti di X secolo attestano

Seminario di Napoli (Contributo alla Storia Napoletana del Cinquecento), Fausto Fiorentino Editore, Napoli, 1957, pp. 27-28. Di DE MAIO si vedano anche 1'articolo Il cardinale Giulio Santori e la riforma del capitolo napoletano nel sec. XVI, "Asprenas", VI (1959), pp. 219-230 e il volume Società e vita religiosa a Napoli nell'età moderna (1656-1799), Edizion Scientifiche Italiane, Napoli 1971.

${ }^{40} \mathrm{G}$. SPARANO, Memorie istoriche cit., pp. 212 ss.

${ }^{41}$ Le Costituzioni di Alessandro Carafa furono stampate a Venezia, per Venturino Rossinello a instantia del nobel huomo messer Matheo Morelli, a corredo del commentario approntato da Alberico Oliva, Rituum Archiepiscopalis et Metropolitanae Ecclesiae Neapolitanae interpretatio seu commentum, cum repertorium in se continente communem praxis causarum in utrogue Foro Canonico ct Civili, magnifici reverendi domini Alberici Oliva Neapolitani sacerdotis inter Decretorum Doctores agnifici reverendi domini Alberici Oliva, Neapolitani sacerdotis et inter Decretorum Doctores acutlssimi, nunc primum editum et impressum una cum Constitutionibus Sinodalibus eiusdem Curiae Metropolitanae, Venetiis, MDXLII. Il titolo riproduceva l'edizione scomparsa del 1484: Constitutiones Sinodales quondam reverendissimi in Christo Patris d. domini Ioannis Dei et Apostolicae Sedis gratia Archiepiscopi Neapolitani et eius Capituli. Confirmatae per reverendissimum d. dominum Alexandrum Caraffam, nunc Archiepiscopum Neapolitanum, et eius Capitulum.

${ }^{42}$ Che Orsini, con la nuova denominazione ufficiale di "Hebdomadarii", intendesse indicare coloro che nelle Costituzioni del 1334 compaiono come "magistri scholarum" lo dimostrano anche le più tarde parole del cardinale Alfonso Carafa, quando nel febbraio del 1565 così stabiliva: "Mandamus venerabililibus Hebdomadariis, Ecclesiae nostrae magistris scholarum nuncupatis, ut iuxta onus sibi iniunctum grammaticam, tantum seu musicam, ac caerimonias ecclesiasticas, clericos eiusdem Ecclesiae nostrae doceant, ac in timore Domini erudiant et instituant, sub poena suspensionis a fructibus huiusmodi benefici seu scholasteriae aut alia, nostro arbitrio imponenda et applicanda". Per la trascrizione di questo passo e la sua contestualizzazione si veda R. DE MAIO, Le origini del Seminario cit., pp. 28-29.

${ }^{43}$ G. SPARANO, Memorie istoriche cit., p. 224. 
l'esistenza di una "congregatio chartulae ecclesiae Stephaniae", tre documenti di XI secolo e tre di XII secolo attestano, il primo, l'esistenza di una "congregatio et ecclesia Sanctae Restitutae virginis et martyris intus episcopium Sanctae Neapolitanae Ecclesiae", i secondi, l'esistenza di più "congregationes sacerdotum et clericorum salutiferae catholicae ecclesiae Sanctae Restitutae de intus episcopio Sanctae Neapolitanae Ecclesiae" (a questi ultimi vanno aggiunti i documenti del 1212, del 1309 e del 1332). Abbiamo infine il documento del 1213 che attesta l'esistenza di una "congregatio Salvatoris", diversa dal Capitolo che qui, dopo le attestazioni degli anni 1167, 1177, 1183 e prima di quella del 1255, è apertamente citato come tale. Nessun accenno agli Ebdomadari vi è nelle Costituzioni liturgiche di D’Ormont del 1317 e in quelle funerarie di Orsini del 1334: essi fanno la loro comparsa con questa denominazione soltanto nelle Costituzioni orsiniane del 1337. Prima di analizzare i dati che emergono da questi documenti, è bene però soffermarsi rapidamente su come essi siano stati fino ad oggi interpretati e sulla base di quali considerazioni pregiudiziali se ne sia data spiegazione.

\section{FALSIFICAZIONI E FRAINTENDIMENTI}

Nel corso dell'intero Novecento e fino all'inizio del terzo millennio, gli storici dell'arte, dell'architettura e della topografia napoletane hanno sostenuto che, prima della fondazione della cattedrale angioina, due chiese cattedrali si ergevano parallele nell'area dell'episcopato. Tale ipotesi sulla coesistenza di due cattedrali non si basa però su osservazioni archeologiche o su testimonianze documentarie: essa è in realtà, come di recente ho scoperto, un'invenzione settecentesca, nata nel contesto di una controversia di natura giuridica sviluppatasi proprio all'interno dell'episcopato napoleta$\mathrm{no}^{44}$.

Quando nel 1713 la Camera Apostolica emanò una sentenza nella quale si vietava agli Ebdomadari della Cattedrale di Napoli di innalzare la croce della medesima cattedrale nei funerali a pagamento di estranei da celebrarsi in altre parrocchie cittadine, questo divieto venne accolto con riluttanza dagli Ebdomadari, in quanto, se effettivamente messo in atto, li avrebbe privati di sostanziose rendite pecuniarie ${ }^{45}$. Essi allora si rivolsero ripetutamente alla Sacra Rota per far accogliere le proprie inopportune richieste, ma il tribunale romano sancì, senza possibilità di appello, che il

\footnotetext{
${ }^{44}$ Per l'individuazione delle ragioni che condussero all'invenzione delle due cattedrali, la discussione dei testi settecenteschi, la disamina delle fonti medievali utilizzate capziosamente per comprovare la falsa teoria, rimando al mio saggio L'invenzione di una tradizione storiografica: le due cattedrali di Napoli, "Prospettiva", 113-114 (2004), pp. 2-31.

${ }^{45}$ Una dettagliata ricostruzione di questi fatti si legge in Agnello FRANCHINI Ragioni per l'illustrissimo e reverendissimo Capitolo della Metropolitana Chiesa di Napoli contro alle pretensioni dei reverendi Eddomadari della medesima, s.n., s.1. [1751]; Memorie intorno al sito della Chiesa Cattedrale di Napoli ed all'essere stata sempre una, con varie notizie critiche sulla qualità ed ufficiatura de' suoi sacri ministri, colle risposte alle soggezioni di monsignor sulla qualita ed ufficiatura de suoi sacri ministri, colle risposte alle soggezioni
Giuseppe Simonio Assemani, appresso Domenico Rosselli, Napoli, MDĆCIV.
} 
privilegio di elevare la croce della cattedrale spettava unicamente al Capitolo dei Canonici e non poteva essere da questi ad altri trasferito. Gli Ebdomadari non appartenevano di certo al Capitolo e "ne in una eademque ecclesia detur biceps corpus distinctum, quod esset summa monstruositas" ${ }^{46}$. Insofferenti della loro condizione subordinata oltre che della loro annosa povertà, desiderosi di privilegi appartenenti per diritto unicamente ai Canonici, gli Ebdomadari non trovarono allora altro modo per rivendicare pari autorevolezza e potere che inventare una tradizione nella quale essi avrebbero costituito il capitolo di una seconda cattedrale napoletana, la Cattedrale del Salvatore o Stefania, un tempo affiancata a quella di Santa Restituta e poi andata completamente distrutta ${ }^{47}$.

In tal modo, pur privati dell'edificio nel quale, millantando, dicevano di aver svolto funzioni capitolari dalla fine del V alla fine del XIII secolo, gli Ebdomadari si ritennero, arbitrariamente, legittimati a portare da soli la croce

\footnotetext{
${ }^{46}$ Sulle decisioni della Sacra Rota e i testi delle sentenze si veda Damiano Romano, Per la insigne Collegiata di San Giovanni Maggiore contro de' RR. Ebdomadari della Cattedrale di questa città, nella Stamperia di Gennaro Migliaccio, Napoli, MDCCLXXII. Per la citazione questa citta, nella Stamperia di Gennaro Migliaccio, Napoli, MDCCL
tra virgolette nel testo cfr. G. SPARANO, Memorie istoriche cit., p. 21.

${ }^{47}$ Sebbene fossero secoli che gli Ebdomadari tentassero in ogni maniera di pretendere privilegi economici e simbolici (lunga, ad esempio, fu la polemica sulle vesti da indossare nelle cerimonie ufficiali), la causa scatenante della controversia fu l'istituzione della Collegiata di San Giovanni Maggiore, avvenuta per decreto di papa Innocenzo XII nel 1692. Il nuovo e potente organismo ecclesiastico costitui per gli Ebdomadari un nemico peggiore persino del Capitolo della Cattedrale, in quanto se questo non aveva mostrato alcun interesse per i loro traffici della Cattedrale, in quanto se questo non aveva mostrato alcun interesse per i loro traffici della Cattedrale, ottenendo nel 17t 3 una sentenza dall'Uditore della Camera Apostolica, nella quale si vietava agli Ebdomadari di innalzare la croce della Cattedrale nei funerali di altre parrocchie (e quindi anche in quella di San Giovanni Maggiore), senza la presenza del Capitolo. Per un preciso resoconto degli scabrosi episodi che precedettero la sentenza romana si vedano le Constitutiones Capituli Sanctae Metropolitanae Ecclesiae Neapolitanae. Francisci Pignatelli auctoritate editae, typis Novelli de Bonis, Neapoli, 1712, in part. pp. 210 ss. La supplica che in quell'occasione ty.j in quell occasione gli Ebdomadari rivolsero al Capitolo cattedrale, per chiedere il suo appoggio nella causa che li opponeva alla Collegiata di San Giovanni Maggiore, rievoca in qualche modo il tono sottomesso dei "confratres Salvatoris", che emerge dal documento dell'arcivescovo Anselmo del 1213, più sopra commentato. Sulla disputa ingaggiata dagli Ebdomadari nel primo Cinquecento per avere vesti analoghe a quelle dei Canonici "la cappa pavonazza" e soprattutto "il rocchetto bianco con busto e maniche"), si veda la descrizione che ne fa l'anonimo autore delle Memorie per difesa dell' illustrissimo e reverendissimo Capitolo de 'Canonici della Chiesa Metropolitana di Napoli, s.n., s.1., [Napoli, 1739]. Infine, sulle precipue motivazioni che indussero gli Ebdomadari a localizzare la cattedrale distrutta proprio al di sotto del transetto de nuovo edificio angioino, sia in riferimento all'esistenza di una fossa comune loro concessa nel 1414 (si veda la plu tarda lastra tombale recante questa data, rimasta fino al 1744 nel pavimento del coro e ora affissa nel cortile del Palazzo Arcivescovile), sia in relazione all'antica sistemazione degli altari, delle tombe e delle reliquie dei santi patroni di Napoli, si veda Nicolò Carminio Falcone, L'intera storia della famiglia, vita, miracoli, traslazioni e culto del glorioso Carminio Falcone, Lintera storia della famiglia, vita, miracoli, traslazioni e culto del glorioso
martire $S$. Gennaro, vescovo di Benevento, cittadino e principal protettore di Napoli, nella Stamperia di Felice. Mosca, Napoli, 1713, pp. 501-502, il primo, a mia conoscenza, a formulare una compiuta ipotesi topografica. Le medesime argomentazioni di Falcone furono poi più volte riprese, doviziosamente spiegate e accresciute di ulteriori particolari, nel corso dell'intera controversia giudiziaria man mano che essa si faceva più accesa: cfr. Memorie in difesa dell'onore dell'illustrissimo e reverendissimo Capitolo metropolitano di Napoli e della giustizia delle causa degli Eddomadari contro la Collegiata di San Giovanni Maggiore, ed in rischiarimento dell'antico e moderno stato della stessa Chiesa metropolitana contro gli errori di altre false memorie stampate per supposta difesa del medesimo e reverendissimo Capitolo per Antonio de' Rossi nella strada del Senato Romano in Roma, 1740, il cui anonimo per Antonio. redattore (poi identificato con Gennaro Majelli da Gaetano MELZI, Dizionario di opere anonime e pseudonme di scrittori italiani o come che sia aventi relazione all italia, 3 voll., col torch di Luigi Di Giacomo Pirola, Milano, 1848-1859, in part. II, p. 183), a dispetto delle dichiarazioni del titolo, usò tutte le armi in suo possesso per schierarsi violentemente contro il Capitolo della Cattedrale.
} 
della Cattedrale nei funerali a pagamento di altre parrocchie cittadine o in altre pubbliche processioni, laddove invece esclusivamente la croce della Cattedrale affidata ai Canonici era autorizzata ad esser sollevata ${ }^{48}$. In quell'occasione gli Ebdomadari ingaggiarono contro i Canonici una lotta senza esclusione di colpi e fecero redigere un folto gruppo di memoriali e relazioni, manoscritte o pubblicate a stampa, di processo in processo presentate alla Sacra Rota romana a difesa della propria indifendibile posizione, basata sulla presunta esistenza di due corpi canonicali perfettamente omologhi e connessi a due antiche cattedrali ${ }^{49}$.

A comprovare la teoria delle due cattedrali e di due capitoli di pari diritti e poteri, screditata ripetutamente dalla Sacra Rota come falsa e menzognera, eppure ogni volta riproposta con maggior forza dagli Ebdomadari che non si rassegnavano a perdere i privilegi pecuniari dei quali il Capitolo stesso li aveva in qualche modo investiti, alcuni dei difensori degli Ebdomadari avanzarono anche un'inedita ipotesi sull'antica topografia medievale dell'area dell'episcopato. La fantasiosa topografia così artificialmente prodotta ebbe tale fortuna che gli studiosi tardo-ottocenteschi e novecenteschi la accettarono come veritiera, dimenticando o ignorando del tutto l'origine della sua creazione e contribuendo a diffondere capillarmente negli studi storicoartistici la tradizione inventata delle due cattedrali. A sostanziarla con le armi del disegno era peraltro intervenuto, nel 1745, un testo che apparentemente con la controversia giuridica non aveva a che fare: il Discorso istorico della Cappella de' Signori Minutoli col titolo di S. Pietro apostolo e di S. Anastasia martire, dentro il Duomo napoletano, di Benedetto Sersale ${ }^{50}$. Nel volumetto, finalizzato a rendere omaggio alla famiglia Minutolo che da secoli aveva il patronato della Cappella di San Pietro nel transetto destro della Cattedrale, apparvero due dettagliate incisioni nelle quali l'autore aveva fatto rappresenta-

${ }^{48}$ Sulla questione dell'uso della croce del Capitolo, che più volte fu negato agli Ebdomadari dalle decisioni rotali, in sentenze alle quali fu impartito il Regio exequatur, si legga il commento alle medesime decisioni in D. ROMANO, Per la insigne Collegiata cit., decreto rotale numero 79: "Nullo modo $[\ldots]$ Hebdomadariis competere valeat supradicta praeminentia sive praerogativa elevandi ac deferendi crucem exclusive ad parochos sive alias ecclesias parochiales unice de iure et vigore dictarum constitutionum sinodalium, reservata soli Capitulo Ecclesiae Metropolitanae, ratione illius superioritatis seu habitualis iurisditionis episcopalis, qua idem Capitulum potitur supra omnes ecclesias civitatis et diocesis".

${ }^{49}$ Tra tutte la più arguta e persino ironica fu quella di Stefano PATRIZI, Relazione dell'antica liturgia della Chiesa di Napoli intorna alla istituzione del Collegio degli Eddomadari di Stefano Patrizi avvocato napoletano al santissimo e beatissimo padre signor nostro Benedetto XIV pontefice massimo, s.n., Napoli, MDCCLI.

${ }^{50}$ Tra i più che numerosi testi pubblicati durante lo svolgimento della causa che vide gli uni contro gli altri prima gli Ebdomadari e i membri della Collegiata i San Giovanni Maggiore, po gli Ebdomadari contro i Canonici del Capitolo cattedrale, l'unico che fu dotato di iflustrazion che descrivessero efficacemente la teoria degli Ebdomadari sulle due cattedrali fu proprio il volumetto di Sersale (edito a Napoli, nella Stamperia di Gianfrancesco Paci), che ebbe cos tanta fortuna che, dopo la morte dell'autore se ne fece persino una riedizione aggiornata (Discorso historico intorno alla Cappella de' Signori Minutoli l.... In questa seconda edizione corretto ed accresciuto cosi delle postille lasciate abbozzate dall'nutore sulla prima che di corretto ed accresciuto cosi delle postille lasciate abbozzate dall autore sulla prima che di alcune altre giunte utili e necessarie, nella Stamperia Raimondiana, Napoli, MDCCLXXVIII). La presenza delle due belle incisioni fu, a mio parere, determinante per la diffusione della teoria: la messa in scena le diede una pubblicità inaspettata. A decretare la fortuna di Sersale è stata però la storiografia del Novecento, alla quale sfuggiva ormai la sua reale collocazione all'interno della mole di pubblicazioni prodotte dalla controversia giuridica settecentesca. 
re dettagliatamente la teoria degli Ebdomadari. In un'improbabile ricostruzione del complesso episcopale, le due cattedrali si stagliavano parallele: l'una rivolta verso la Platea Capuana, oggi Via dei Tribunali, l'altra rivolta verso la Somma Piazza, dove si ergeva anche la chiesa trecentesca di Donnaregina (fig. 4).

La controversia funeraria dall'ambito strettamente giuridico si era ormai spostata verso altri lidi, latamente eruditi: intervennero a prendere posizione prestigiosi accademici del tempo, come il Mazzocchi, già molto celebre e ormai alla fine della sua lunga carriera ${ }^{51}$ o Giuseppe Simonio Assemani, l'illustre "primo custode" della Vaticana ${ }^{52}$, per non citarne che alcuni. Essa continuò in forme analoghe per tutta la seconda metà del Settecento, riproponendo stancamente le medesime argomentazioni messe in campo dai primi apologisti degli Ebdomadari. A sancire inesorabilmente la vittoria definitiva della falsa tesi degli Ebdomadari fu però un erudito ottocentesco noto per la sua acribia filologica, Bartolommeo Capasso, che pubblicò, commentandoli, alcuni dei documenti relativi alle congregazioni dell'episcopio napoletano e ne dedusse, facendo sua l'ipotesi delle due cattedrali, che le congregazioni menzionate in quei documenti dovevano necessariamente riferirsi a due diverse cattedrali: "In nostris enim monumentis congregationis ecclesiae Stephaniae simul ac congregationis ecclesiae S. Restitutae notitia occurrit. Duplicem ergo cathedralem duplicemque in ea clerum hisce temporibus Neapoli extitisse non dubito. Quomodo autem utraeque congregationes in unum coaluerint corpus et congregatio $\mathrm{S}$. Restitutae superiorem, congregatio Stephaniae vero inferiorem locum obtinuerint, nescio. Certe ex quadam nova archiepiscopalis capituli constitutione, cum vetus Stephania obsolesceret, vel praesens cathedralis exaedificaretur, id effluxisse dicendum est" 53 .

Non molti anni dopo, nella sua Pianta della città di Napoli, ben nota agli studiosi di topografia cittadina, Capasso ritornava sulla questione delle due cattedrali, le descriveva con l'ausilio dell'immaginazione e così concludeva: "Ciascuna di queste basiliche che abbiamo descritte aveva il proprio clero, poiché nei documenti del tempo s'incontrano spesso da una parte la congrega-

\footnotetext{
${ }^{51}$ Cfr. R. DE MAIO, Alessio Simmaco Mazzocchi e la filologia del Settecento, in "Critica Storica", n.s., X, 1973; Alessio Simmaco Mazzocchi e il Settecento meridionale, a cura di Pietro BORRARO, Palladio editrice, Salerno, 1979. Non escludo che mulla svalutazione della Dissertatio historica di Mazzocchi sia in qualche modo pesato il giudizio che dell'erudito diede Johann Joachim Winckelmann in una lettera del 1758, definendolo "rimbambito": per una disamina di questa e di altre lettere del Winckelmann riguardanti l'ambiente accademico napoletano, cfr. R. DE MAIO, Società e vita religiosa cit., pp. 299-230 e note corrispondenti.

${ }^{52}$ Contro le asserzioni pronunciate da Assemani nelle ottanta pagine del saggio Edict perentorii repulsa qua sententia de duabus diversis Basilici Neapolitanis, Constantiniana et Stephania defenditur [...], accluso come introduzione al III volume dei suoi Italicae historiae scriptores ex Bibliothecae Vaticanae aliarumque insignium bibliothecarum manuscriptis codicibus, 4 voll., ex Typographia Linguarum Orientalium Angeli Rotili et Philippi Bacchelli Romae, 1751-52, Mazzocchi aggiunse alla sua Dissertatio un Editto perentorio, nel quale s difendeva dalle accuse mossegli dall'erudito romano. Le vere ragioni per le quali Assemani si schierò dalla parte degli Ebdomadari sono ancora da indagare.

${ }^{53}$ B. CAPASSO, Monumenta ad Neapolitani Ducatus historiam pertinentia, ex Regio Typographeo Equ. Francisci Giannini, Neapoli, II/1, 1885, p. 31 nota 2.
} 
tio sacerdotum et clericorum salutifere ecclesie S. Restitute, col primicerio, l'arcidiacono, i preti cardinali, i diaconi e suddiaconi, dall'altra la congregatio sacerdotum et clericorum ecclesie Stephanie, della quale era a capo un archipresbiter et cimiliarca" ${ }^{54}$

Proprio l'autorevolezza di Capasso e il ruolo intellettuale che egli svolse nella Napoli di fine Ottocento fecero sì che la sua ricostruzione del sito episcopale, modellata, con qualche variazione, sulle incisioni di Sersale, risultasse a partire da quel momento l'unica ipotesi praticabile, tanto che persino Ėmile Bertaux la accolse senza la minima esitazione nei suoi volumi sull'arte del Medioevo meridionale ${ }^{55}$. Da allora in poi, anche grazie ai numerosi interventi di Monsignor Strazzullo che sposò la tesi delle due cattedrali con grande entusiasmo ${ }^{56}$, la storiografia novecentesca ha continuato a spiegare i documenti sulle congregazioni utilizzando la teoria delle due cattedrali, mentre a sua volta chi ha voluto giustificare questa teoria ha tautologicamente chiamato in causa proprio quei documenti.

\footnotetext{
${ }^{54}$ Il saggio fu pubblicato in "Archivio storico per le province napoletane”, XVII, 1892, pp 422-484 in part. per la citazione p. 466 e note corrispondenti) e fu riedito in Topografia della città di Napoli nell'XI secolo, s.n., Napoli, 1895. La proposta di Capasso di individuare la citta di Napoli nell'XI secolo, s.n., Napoli, 1895. La proposta di Capasso di individuare la ragionamento sulla posizione dello xenodochium fondato dal vescovo Atanasio I alla fine de IX secolo, attestato nella Vita sancti Athanasii episcopi (cfr. infra, nota 59). Malgrado che la topografia storica sia considerata "il campo in cui Capasso espresse al meglio la sua originalità e conseguì i risultati maggiori", soprattutto per la capacità di introdurre "obiettivi e metodi del tutto nuovi rigorosamente scientifici, dentro la plurisecolare fortuna di un genere letterario, quello delle descrizioni e guide storico-monumentali della città" (Mario DEL TREPPO, erudizione nella Sapoli dell'Ottocento a cura di Giovanni Vmeo Capasso. Storia, flologia, erudizione nella Napoli dell Ottocento, a cura di Giovanni VITOLO, Guida, Napoli, 2005, pp 15-131, in part. pp. 61-62 per le citazioni tra virgolette), e malgrado il giudizio assai positivo che delle ricostruzioni topografiche di Capasso diede già Benedetto Croce (Un innamorato di Napoli. Carlo Celano "Napoli Nobilissima", II (1893), pp. 65-70), è necessario riconoscere che le conclusioni di Capasso sulla Cattedrale di Napoli furono piuttosto approssimative. Senza entrare nel merito dell'interpretazione che egli diede delle fonti medievali e della storiografia napoletana cinque-seicentesca relative alla Cattedrale, sulla quale troppo ampio sarebbe il discorso e che rimando ad altra occasione, un caso mi pare veramente esemplare del suo metodo: la descrizione della presunta distrutta Stefania. "La Stefania, come il Duomo attuale, aveva l'aspetto o l'ingresso principale ad occidente, l'absida, o la tribuna, ad oriente, ed era quasi nello stesso modo come ora, inquadrata da quattro torri alte e acuminate (procero cacumine), due delle quali nella parte postica sono accertate dalla testimonianza di Giovan Diacono ed altre due nella parte anteriore si possono per ragioni di euritmia fondatamente congetturare". La descrizione di Capasso, fondata su una lettura imprecisa delle testimonianze medievali (ad esempio, Giovanni Diacono non dice affatto che le due torri si trovavano nella parte posteriore), e su assunti alquanto avventati ("per ragioni di euritmia"), per nulla parte posteriore), e su assunti alquanto avventati ("per ragioni di euritmia"), per nulla costantemente e acriticamente riproposta nel corso dell' intero Novecento. Sulla questione della posizione delle torri nella Cattedrale di Napoli al tempo del vescovo Stefano II, al quale si riferisce il passo dei Gesta episcoporum Neapolitanorum citato da Capasso, rinvio al mio intervento L'architettura della Cattedrale di Napoli nell' Altomedievo, tra potere ducale e potere episcopale, tenuto al Convegno internazionale Elites and architecture in the Middle Ages (International Reasearch Center for Late Antiquity and Middle Ages, Motovun, University of Zagreb, 1- 4 giugno 2006), i cui atti sono in corso di pubblicazione.

${ }^{55}$ Ėmile BERTAUX, L'art dans l'Italie méridionale, 3 voll., A. Fontemoing, Paris 1903, in part. I, p. 30.

${ }^{56}$ Oltre ai saggi citati supra, nota 9, si veda anche F. STRAZZULLO, Le due antiche cattedrali di Napoti, "Campania sacra”, V'(1973), pp. 177-241.
} 


\section{NUOVE PROPOSTE}

A Napoli non sono mai esistite due cattedrali e l'unico edificio con funzioni episcopali, antecedente l'edificazione della cattedrale gotica, fu dedicato al Salvatore, fu denominato "Stefania" dal nome di uno dei suoi vescovi committenti, e fu intitolato a Santa Restituta solo alla fine del Duecento, in concomitanza con la dedicazione all'Assunta della nuova cattedrale angioina ${ }^{57}$. Prima che gli Ebdomadari avanzassero la loro falsa asserzione sull'esistenza di due cattedrali parallele, nessuno a Napoli o fuori di Napoli aveva dichiarato, sostenuto o semplicemente ipotizzato, che le cattedrali fossero due. Non vi è traccia di una seconda cattedrale nei Gesta episcoporum Neapolitanorum, la cronaca dei vescovi di Napoli che costituisce la fonte cardine per l'Altomedioevo napoletano, né ve ne è nelle fonti storiche medievali non locali che ricordano la città, come i Chronica minora di Beda, il Chronicon in aetatem sex divisum di Adone, vescovo di Vienne, o il Chronicon Vulturnense, dove la cattedrale è sempre una ed una sola. Né infine ve ne è traccia nella gran messe di documenti di età angioina relativi alle donazioni reali per la nuova costruzione episcopale, o nelle fonti trecentesche quali il Chronicon di Santa Maria del Principio o la cosiddetta Cronaca di Partenope, e meno che mai nella vasta guidistica locale cinque-seicentesca o nella storiografia sei-settecentesca del Regno e della Chiesa di Napoli58.

La teoria delle due cattedrali non è altro che una storia inventata, per ragioni puramente pecuniarie, da un corpo di mansionari inferiori (non privi di potere e di appoggi), ai quali era venuta a mancare la loro principale fonte di sostentamento materiale. Pur vantandosi di discendere da un collegio sacerdotale fondato dal vescovo Atanasio I nel corso del IX secolo ${ }^{59}$, gli

\footnotetext{
${ }^{57}$ Su questo argomento ho tenuto una relazione, dal titolo Santa Restituta venuta dall'Africa: l'utilizzazione canonicale di un mito altomedievale nella Napoli angioina, al Convegno internazionale I Santi venuti dal mare (Bari-Brindisi, 14-17 dicembre 2005), i cui atti sono in corso di pubblicazione. 44.

${ }^{58}$ Per la disamina del dettato di queste fonti rimando al mio articolo citato supra, in nota

${ }^{59}$ Nella Vita sancti Athanasii (Vita et Translatio s. Athanasii (BHL 735 e 737). Sec. IX, a cura di Antonio VUOLO, Istituto Storico Italiano per il Medioevo, Roma, 2001, p. 128), un testo pressoché contemporaneo alla sezione dei Gesta episcoporum Neapolitanorum redatta dall'agiografo Giovanni Diacono, si legge che il vescovo "zelo fretus divino, constituit sacerdotes hebdomadarios in ecclesia Domini Salvatoris, quae Stephania vocatur, qui in ea continuis diebus publicam missam celebrarent, sicut mos est Romanae Ecclesie [...]. In ecclesiis quoque Beati Andreae Apostoli et Protomartyris custodem deligavit, ubi et res optulit ad luminariorum concinnationes et sumptus ipsius custodis. Ecclesiae vero.Sanctae Restitutae [..; connectit utrosque Iohannem Baptistam scilicet et precursorem Domini atque Evangelistam; Il passo è stato interpretato dagli apologisti degli Ebdomadari come la prova che non solo la loro origine risalisse al prestigioso santo-vescovo Atanasio, ma che la chiesa di Santa Restituta era un edificio distinto dalla basilica del Salvatore o Stefania. Il lessico usato dal redattore della Vita Athanasii, la so unza e la natura dei fatti narrati rendono invece evidente che siamo di fita Athanasil, la sequenza e la natura dei fatti narrati rendono invece evidente che siamo di fronte all'elencazione di una serie di cappelle ("ecclesiae") che si trovavano all'interno della Cattedrale del Salvatore o Stefania. Ritengo si trattasse verosimilmente di strutture di dimensioni modeste, dotate di altari dedicati ai medesimi santi che ne stabilivano la titolazione, probabilmente connesse con il modello romano di San Pietro in Vaticano e di San Giovanni in Laterano, dove i papi Ilaro e Simmaco avevano allestito tre cappelle, connesse tra di loro, dedicate ai santi Giovanni Battista, Giovanni Evangelista e alla Croce (sul caso di Roma cfr.
} 
Ebdomadari non fecero la loro pubblica comparsa con questo titolo prima del $1337^{60}$. Considerato dunque che non sono mai esistite due cattedrali parallele (una intitolata al Salvatore ed una a Santa Restituta), ma che i due titoli si successero l'uno all'altro in quel delicato momento di ricostruzione delle memorie della Chiesa locale che accompagnò l'edificazione della nuova cattedrale patrocinata dalla famiglia reale angioina; tenuto conto che è verosimile che la vecchia Cattedrale del Salvatore assumesse il nome di Santa Restituta proprio nel momento in cui fu affidata ai Canonici del Capitolo che quel titolo comunque già detenevano (e ciò dové avvenire senz'altro prima del 1309 , come testimonia il documento di Siginolfo nel quale si dice che i Canonici del Capitolo si fregiavano appunto del titolo di Santa Restituta), non resta che riflettere a questo punto sui documenti medievali, più sopra riportati, facenti riferimento alle congregazioni della Cattedrale e provare ad avanzare una nuova ipotesi sulla loro identificazione.

Chi se ne è occupato nel corso del Novecento, convinto che fossero esistite due cattedrali, la Stefania e Santa Restituta, ha proposto: a- che nella "congregatio chartulae ecclesiae Stephaniae", che ricorre nei documenti del 932 e del 977, si debba identificare il collegio dei chierici e dei sacerdoti della Cattedrale del Salvatore, detta anche Stefania; b- che questo medesimo collegio della Stefania sia da riconoscersi anche nella "congregatio Salvatoris", attestata nel documento dell'arcivescovo Anselmo del 1213; c- che nei "confratres Salvatoris"del 1213 siano da identificarsi gli Ebdomadari della Costituzione di Orsini del 1337. Viceversa, nella "congregatio Sancte Restitutae", attestata nei documenti del 1066, 1100, 1146, 1150, 1188, andrebbe riconosciuto il clero addetto alla seconda delle due cattedrali, quella di Santa Restituta, a sua volta considerato distinto dal vero e proprio Capitolo metropolitano ${ }^{61}$.

Sono d'accordo sul primo punto: con la definizione "congregatio chartulae ecclesiae Stephaniae" si vuole probabilmente segnalare in quei due documenti la congregazione sacerdotale facente capo alla Cattedrale napoletana e la si indica nel modo più semplice, definendola con il nome con cui la Cattedrale del Salvatore era più di frequente chiamata in città.

Sible DE BLAAUW, Cultus ed decor. Liturgia e architettura nella Roma tardo-antica e medievale, Biblioteca Apostolica Vaticana, Città del Vaticano, 1994, II, p. 489). Per quanto riguarda invece un altro passo della Vita Athanasii ampiamente utilizzato dagli Ebdomadari (mi riguarda invece un altro passo della Vita Athanasii ampiamente utilizzato dagli Ebdomadari (mit riferisco all'espressione "binas praesulum [...] sedes, ad instar duorum Testamentorum": Vita
et Translatio cit., p. 117) nel quale propongo di riconoscere la Cattedrale cittadina del et Translatio cit., p. 117), nel quale propongo di riconoscere la Cattedrale cittadina del
Salvatore o Stefania e la basilica extramuranea di San Gennaro, rinvio al mio intervento Tombe Salvatore o Stefania e la basilica extramuranea di San Gennaro, rinvio al mio intervento Tombe di re, vescovi e santi nella Cattedrale di Napoli: memoria liturgica e memoria profana, tenuto settembre 2005), i cui atti sono in corso di pubblicazione.

${ }^{60} \mathrm{~L}$ 'unica ambigua attestazione che precede tale data si trova in un documento dell'inizio del XII secolo relativo ad un possedimento del monastero dei Santi Severino e Sossio, nel quale si menziona un presbitero, "cui nomen Iacobus, ebomadarius Stephaniae", "ma nulla prova che si tratti di un membro del collegio che nel Trecento assume il nome di "Ebdomadari": cfr. $L$ 'antico inventario delle pergamene cit., II, p. 805 [698].

${ }^{61} \mathrm{C}$. D. FONSECA, "Congregationes clericorum et sacerdotum» a Napoli nei secoli XI e XII, "Aevum", XXXIV'(1960), pp. 104-121, che a tutt'oggi resta il solo riferimento su tale controversa questione. 
È invece plausibile, alla luce delle nuove acquisizioni, che nella "congregatio Sanctae Restitutae" si possa riconoscere una forma originaria del Capitolo della medesima Cattedrale del Salvatore. La denominazione in omaggio alla santa martire Restituta deve esser nata, a mio parere, proprio nel corso dell'XI secolo, sulla base del particolare legame esistente tra la congregazione di chierici della Cattedrale del Salvatore e l'altare nel quale si conservavano le spoglie di Santa Restituta. Questo altare doveva già trovarsi laddove è tuttora visibile il moderno ricettacolo delle reliquie della santa, ovvero all'interno della cappella che nel primo Trecento risulta dedicata a Santa Maria del Principio e che dové assumere il nuovo titolo in connessione con la realizzazione in essa del mosaico raffigurante la Madonna con il Bambino tra san Gennaro e santa Restituta ${ }^{62}$, datato da un'iscrizione al $1313^{63}$.

Che tale congregazione di Santa Restituta, documentata per la prima volta nel 1066, sia in tutto diversa e distinta dal collegio di mansionari noti fin dall'inizio del XIII secolo come "congregatio Salvatoris", e che questi ultimi a loro volta non abbiano nulla a che fare con la "congregatio chartulae ecclesiae Stephaniae" di X secolo, lo dimostra innanzitutto la bolla del gennaio 1213, nella quale è appunto attestata per la prima volta la presenza di una "congregatio Salvatoris". Dal documento si evince con chiarezza che i "confratres Salvatoris" si trovano ad un livello gerarchico nettamente inferiore a quello dei Canonici del Capitolo, posizione che impedisce di credere che si trattasse di un altro Capitolo ${ }^{64}$. Non si dimentichi infatti che l'esenzione dalle collette, alla quale più sopra abbiamo accennato, non fu affatto un privilegio, ma semplicemente una concessione data dall'arcivescovo Anselmo, e per giunta su richiesta dello stesso Capitolo che evidentemente aveva interesse che i "confratres Salvatoris" continuassero a svolgere i loro compiti subordinati.

Se questa "congregatio Salvatoris", che fa la sua prima apparizione proprio nel documento del 1213, avesse costituito il legittimo Capitolo di una cattedrale ancora in piedi (si tenga conto che neanche gli Ebdomadari e i loro apologisti pensavano che a quella data la Cattedrale del Salvatore fosse già

${ }^{62} \mathrm{La}$ Cappella di Santa Maria del Principio, nella quale ho motivo di riconoscere l'originaria Cappella di Santa Restituta in cui il clero napoletano si compiaceva di vedere l'espressione della fondazione costantiniana della Cattedrale di Napoli, è cosi denominata per la prima volta nel Chronicon di Santa Maria del Principio, databile all' inizio del secondo decennio del Trecento. E verosimile che alla redazione del Chronicon si accompagno la realizzazione del mosaico raffigurante la Vergine con il Bambino in trono, tra san Gennaro e santa Restituta, che un'iscrizione contemporanea dichiara restauro di un' immagine preesistente di età costantiniana. Nel medesimo Chronicon si sostiene che anche il Capitolo cattedrale fu fondato da Costantino. Tale mitica fondazione ben si inquadrava nell'ambito di un generale recupero delle radici della Chiesa di Napoli. Su questo tema si leggano le pertinenti osservazioni di Domenico MALLARDO, Cimiliarchio e cimiliarchi della Chiesa di Napoli fino al sec. XIV, "Asprenas", V (1958), pp. 47-70.

${ }^{63} \mathrm{Per}$ la formulazione di questa ipotesi di datazione, contro la proposta corrente che vuole datare il mosaico, al 1322, si veda la mia recensione al volume Il Duomo di Napoli dal paleocristiano all'età angioina cit., "Napoli Nobilissima", V serie, V (2004), pp. 74-77.

${ }^{64}$ Che essi siano presieduti dal Cimiliarca, non deve meravigliare. Questa figura, che compare per la prima volta nel documento del 932 (cfr. supra, nota 17), aveva un duplice ruolo: faceva parte integrante del Capitolo dei Canonici, ma nel contempo controllava e gestiva il collegio degli Ebdomadari: D. MALLARDO, Cimiliarchio e cimiliarchi cit. 
andata distrutta), in nessun modo si spiegherebbe il tono generale del documento, nel quale la congregazione del Salvatore si lamenta della propria povertà e chiede all'arcivescovo una speciale dispensa, in ciò appoggiata dal Capitolo al quale è palesemente subordinata. In base alla teoria delle due cattedrali, la Cattedrale del Salvatore o Stefania sarebbe andata distrutta solo alla fine del XIII secolo, durante i lavori di costruzione del nuovo edificio cattedrale, gotico e di patrocinio angioino, dedicato all'Assunta. Se nel 1213 la vecchia Cattedrale del Salvatore era ancora praticabile, sarebbe paradossale e incongruente che i suoi Canonici si rivolgessero all'arcivescovo per una supplica e si facessero in ciò tutelare dal legittimo Capitolo.

Fermo restando che la Cattedrale era una ed una sola, non resta che ipotizzare che il clero della Chiesa cattedrale di Napoli nel 1213 aveva già assunto quella configurazione che sarà sancita ufficialmente nelle Costituzioni orsiniane del 1337. Ciò significa che i "confratres Salvatoris" non erano altro che i futuri Ebdomadari, una congregazione gerarchicamente sottoposta al Capitolo dei Canonici. Essi però non hanno e non potrebbero avere, per la loro stessa natura, che ben emerge dalla lettura del documento del 1213, nessun legame con la "congregatio chartulae ecclesiae Stephaniae" di X secolo, nella quale può ragionevolmente riconoscersi il clero cattedrale antecedente la formazione del vero e proprio Capitolo.

Quanto alla "congregatio Sanctae Restitutae" e alla sua identificazione, un documento risulta davvero dirimente: la donazione di Bartolomeo Siginolfo, Camerario del Regno. Nel documento del 1309 Siginolfo dona i suoi beni al primicerio, ai diaconi, ai cardinali e a tutti gli altri Canonici della Chiesa metropolitana, formanti la congregazione di Santa Restituta. La nuova cattedrale era ormai già in piedi: il Capitolo dei Canonici conservava il titolo di Santa Restituta che lo aveva contraddistinto negli ultimi tre secoli. Di lì a poco essi avrebbero fatto tutto quanto era nelle loro facoltà e capacità per mantenere intatto ciò che restava della vecchia Cattedrale del Salvatore ${ }^{65}$.

L'importante studio di Gennaro Maria Monti sulle confraternite dell'Italia settentrionale e centrale, edito all'inizio del Novecento ${ }^{66}$, e quelli, altrettanto rilevanti per chi si occupa di queste tematiche, di Gilles Girard Meersseman sulla natura degli istituti associativi del clero, pubblicati negli anni Sessanta e Settanta del secolo scorso ${ }^{67}$, hanno messo in luce con chiarezza la difficoltà di traduzione della diversa terminologia usata nelle fonti

\footnotetext{
${ }^{65} \mathrm{Cfr}$. di chi scrive, L'invenzione di una tradizione cit., pp. 18-20.

${ }^{66} \mathrm{G}$. M. MonTI, Le confraternite medievali dell'alta e media Italia, La Nuova Italia, Venezia, 1927.

${ }^{67}$ In particolare si veda G. G. MEERSSEMAN, Ordo fraternitatis. Confraternite e pietà dei laici nel Medioevo, in collaborazione con Gian Piero Pacini, Herder, Roma, 1977. Sulle "chartule fraternitatis" dei laici un importante contributo è venuto da G. VITOLO, Istituzioni ecclesiastiche e vita religiosa dei laici nel Mezzogiorno medievale. Il codice della confraternita di S. Maria di Montefusco (sec. XII), Herder, Roma, 1982.
} 
archivistiche medievali, ma soprattutto la difficoltà di desumere dalle fonti documentarie una teoria univoca sul ruolo e le funzioni delle congregazioni. $\mathrm{Si}$ tratta di una questione storiografica di grande portata, sulla quale opportunamente attirava l'attenzione Cosimo Damiano Fonseca, già nel 1959 $\mathrm{e}$ più volte nei decenni successivi, rilevando la necessità ineludibile di individuare le precise caratterizzazioni giuridiche di tali istituti.

Gli studi sulla formazione degli istituti canonicali e sul loro sviluppo nei secoli del Medioevo stanno peraltro ricevendo, proprio in questi ultimi anni, un rinnovato impulso, anche metodologico, che non mancherà di far sentire il suo peso ${ }^{68}$. Non ho intenzione qui di entrare nel merito della questione, ben discussa proprio dall'autorevole Fonseca, sulla datazione relativa alla nascita di un Capitolo metropolitano a Napoli ${ }^{69}$, perché tale questione esula dagli scopi del mio intervento. È d'altronde pienamente condivisibile l'individuazione nell'episcopato napoletano, ben prima del XII secolo, di "un clero gerarchicamente strutturato, che abbozza, anche se embrionalmente, un vero e proprio istituto capitolare" ${ }^{70}$. Ritengo però che sia integralmente da rivedere l'ipotesi, finora generalmente condivisa e data per acclarata, che intende identificare nella congregatio Sanctae Restitutae i

${ }^{68}$ Oltre alla voce classica di Charles DEREINE del 1953, Chanoines, nel Dictionnaire d'histoire et de géographie ecclésiastique t. XII coll, 353-405, Georges DESPY, Note sur les
sens de Capitulum, "Archivum Latinitatis Medii Aevii", XX (1950), pp. 245-247, e il colloquio

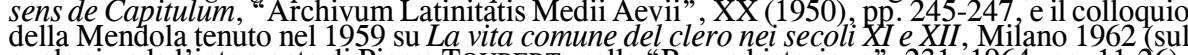
quale si veda l'intervento di Pierre TOUBERT, nella "Revue historique", 231, 1964, pp. 11-26), $\mathrm{mi}$ limito qui a citare solo alcune voci essenziali della seconda metà del Novecento: Charles GIROUD, L'Ordre des chanoines régulièrs de Saint-Augustin et des diverses formes de régime interne. Essai de synthese historico-juridique, Editions du Grand-Saint-Bernard, Martigny, 1961 un testo chiave per la comprensione di questi temi-il più generale Max HEIMBUCHER, Die Orden und Kongregationen der Katholischen Kirche, 2 voll. Verlag Ferdinand Schöningh, München-Padeborn, 1966 , in part. I, pp. 392 ss.; la raccolta di scritti di Jean BECQUET, Vie canoniale en France au Xle et XIIe siecle, Variorum Reprints, London, 1985; la miscellanea canoniale en France au Xle et XIIe siecle, Variorum Reprints, London, 1985; la miscellanea
di studi in Le monde des chanoines (Cahiers de Fanjeaux, 24), Privat, Toulouse, 1989, con utili diferimenti alla bibliografia precedente; le ricerche di Jean CHATILLON, Le mouvement canoniale au Moyen Age: réforme de l'Eglise, spiritualité et culture, a cura di Patrice SICARD, Brepols, Paris, 1992, in part. le pp. 131 ss., sulla spiritualità dell'ordine canonicale. Più d recente, e in ambiti più specificamente monografici, segnalo il volume curato da Mathieu ARNOUX, Des clercs au service de la riforme: etudes et documents sur les chanoines réguliers de la province de Rouen. Brepols, Turnhout 2000; gli Atti della Giornate di Studio tenutesi Dresda nel dicembre del 2000 editi a cura di Gert MELVILLE e Anne MüLLER, Regula Sanct Augustini: normative Grundlage differenter Verbönde im Mittelalter, Augustiner-CularherrenAugustini: normative Grundlage differenter Verbande im Mittelalter, Augustiner-ChorherrenVerlag, Paring 2002, e soprattutto Imponente lavoro di Martin A. CLAUSSEN, The Reform of the Frankish Church: Chrodegang of Metz and the Regula Canonicorum in the Eigh Century, Cambridge University Press, Cambridge, 2004. Interessanti contributi si leggono in Ecrire son histoire. Les communautés régulières face a leur passé, "Actes du 5 e colloque international du Centre européen de recherches sur les congrégations et ordres religieux (SaintEtienne, 6-8 novembre 2002), Publications de 1'Universite de Saint-Etienne, 2005. Utili spunti, e riferimenti bibliografici, si trovano anche alle voci Vita canonicale, nel Dizionario degli Istituti di Perfezione, III, Roma 1978, coll. 240-253; Augustiner-Chorherren, nel Lexicon des Mittelalters, 1, München'1980, coll. 1219-1220; Canonici regolari, in Dizionario enciclopedico del Medioevo, diretto da André VAuCHEZ, Parigi, Roma, Cambridge, 1998, I, pp. 307 ss.

${ }^{69} \mathrm{Su}$ questi temi si veda C. D. FonSECA, Medioevo canonicale (Pubblicazioni dell'Universitâ Cattolica del Sacro Cuore. Contributi. Serie III. Scienze storiche. 12), Vita e pensiero, Milano 1970, in part. pp. 56 ss. sulla storiografia relativa ai movimenti canonicali; IDEM, Canoniche regolari, capitoli cattedrali e «cura animarum», in Pievi e parrocchie in Italia nel basso Medioevo (sec. XIII-XIV). Atti del VI Convegno di Storia della Chiesa in Italia (Firenze, 21-25 settembre 1981), Herder, Roma 1984, pp. 257-278.

${ }^{70}$ C. D. FonSECA, «Congregationes clericorum et sacerdotum» cit., pp. 114-115. 
canonici di una delle due presunte cattedrali napoletane (Santa Restituta), nella congregatio Salvatoris di primo Duecento e nella congregatio ecclesiae Stephaniae di X secolo i canonici della seconda delle due presunte cattedrali, quella del Salvatore o Stefania.

Ė necessario a questo punto degli studi ripensare l'intera questione del clero napoletano prima della codificazione avvenuta in età angioina ad opera di Humbert d'Ormont e soprattutto di Giovanni Orsini. E non sembri strano agli storici della Chiesa di cominciare ad indagare questo tema a partire da una nuova rilettura della topografia e dell'architettura del complesso episcopale napoletano. Paradossalmente fu proprio a causa dei contrasti tra i vari componenti del clero di Napoli che si generò un'invenzione che si è così radicalmente incardinata nella memoria della città che si sta rivelando piuttosto difficile sradicarla, malgrado la documentazione giuridica settecentesca dichiari a gran voce che non sono mai esistite due cattedrali e quindi non sono mai esistiti due Capitoli cattedrali.

Quando nel 1772 il redattore delle Memorie in difesa dell'insigne collegio dei sacri ministri della Cattedrale napoletana, chiamati Eddomadari pubblicò l'incisione con la solenne processione di accoglienza del cardinale Barberini, vi accluse anche una seconda grande carta di tavola, ripiegata in molte parti, raffigurante una "Descrizione della pianta della nuova Cattedrale di Napoli, dell'antica detta Stefania, o S. Salvatore, e della Basilica di S. Restituta" (fig. 5). L'immagine, corredata da un'analitica legenda descrittiva dei singoli siti, aveva la funzione di riprodurre visivamente le due presunte antiche cattedrali, oltre alla nuova cattedrale di patrocinio angioino ancora in funzione e al Palazzo Arcivescovile con i suoi ampi giardini. Le incisioni e l'intero volume costituirono, nell'immediato, la risposta ad un singolo sgradevole episodio che solo pochi mesi prima aveva riaperto la controversia tra i collegi clericali della diocesi ${ }^{71}$, ma in effetti le due belle immagini non

\footnotetext{
${ }^{71}$ Sia la cavalcata solenne di Barberini sia la pianta descrittiva del complessso episcopale prima delle presunte distruzioni medievali furono evidentemente realizzate proprio in occasione del riacutirsi della controversia. La versione dei fatti accaduti il 24 novembre 1771 può leggersi nel documentato e accurato lavoro di Damiano Romano, così come in quello, diversamente orientato e schierato, di Francesco Peccheneda: "Sotto i 24. di Novembre dell'anno scorso [scilicet 1771] gli Eddomadari, associando un cadavero, si portarono alla chiesa di San Giovanni Mago gli Eddomadari, associando un cadavero, si portarono alla chiesa di San Giovanni Maggiore, e senza avere premesso alcuno avviso, pretesero di poter entrare in essa colla croce inalberata, non ostante che il canonico cimiliarca, loro capo, memore della decisione della Sacra Congregazione, avesse ordinato al chierico che la portava che abbassata l'avesse. E perché il chierico, il quale sta al servizio della suddetta Chiesa Collegiata, sapendo molto bene che non potevano essi entrare colla suddetta croçe inalberata, la tolse dall'asta e la consegnò in mano del chierico stesso che la porțavạ. Perciò essendo essi entrati nella sacrestia incominciarono a maltrattare con parole ingiuriose il canonico parroco che in quella $\mathrm{s}$ ritrovava, scagliando le ingiurie anche contro tutt' $i$ Capitolari. Indi per colorire tanti e si enorm attentati proposero querela criminale nella Reverenda Curia Arcivescovile contro del chierico della suddetta Collegiata e contro di uno eddomadario della medesima, caratterizzando l'azione del suddetto chierico come dispreggiativa della croce, quasi fosse stato invasato dallo spirito degl'Iconoclasti, ed accusando il suddetto eddomadario come istigatore della irriverenza secondo essi dicevano, usata alla suddetta croce, ed incriminando lo stesso chierico di aver dati due pugni a colui che la portava: circostanza inventata di sana pianta dagli essi Eddománari, perché non è affatto vera. [Gli Ebdomadari ...] non sono mai stati che semplici fratanzari" (D) ROMANO, Per la insigne Collegiata cit., pp. XLII ss.); "Egli è da sapersi che nel dì 24 novembre dello scorso anno, nel mentre l'insigne collegio dei' sacri ministri della Chiesa cattedrale chiamati Eddomadari associava con la croce inalberata un cadavere che si dovea
} 
erano altro che la faccia spettacolare e speculare di una controversia giuridica che gettava le sue radici altrove e molto lontano nel tempo.

Data di ricezione dell' articolo: luglio 2006.

Data di accettazione e versione finale: agosto 2006.

seppellire nella parrocchiale chiesa di San Giovanni Maggiore, avvenne che dinanzi alla porta di essa chiesa, prima di entrare, si commise un attentato da un prete della chiesa suddetta, don Cristoforo Gambaioli, e da un altro, che serviva qual chierico la stessa chiesa, per nome Vincenzo Silvestri, con istrappare la croce dall'asta, ov'era inalberata, con publica e scandalosa irriverenza" (Memorie in difesa cit., pp. IV ss.).

ANUARIo de Estudios Medievales (AEM), 36/2, julio-diciembre 2006, pp. 613-649. ISSN 0066-5061 